\title{
DISTRIBUTION OF PERIODIC POINTS OF CERTAIN GAUSS SHIFTS WITH INFINITE INVARIANT MEASURE
}

\author{
FLORIN P. BOCA AND MARIA SISKAKI
}

\begin{abstract}
This paper investigates the periodic points of the Gauss type shifts associated to the even continued fraction (Schweiger) and to the backward continued fraction (Rényi). We show that they coincide exactly with two sets of quadratic irrationals that we call $E$-reduced, and respectively $B$-reduced. We prove that these numbers are equidistributed with respect to the (infinite) Lebesgue absolutely continuous invariant measures of the corresponding Gauss shift.
\end{abstract}

\section{INTRODUCTION}

Euclidean algorithms and their associated continued fraction expansions generate interesting examples of (non-invertible) measure preserving transformations, called Gauss shifts. The best known is the Gauss map

$$
T:[0,1) \longrightarrow[0,1), \quad T(x):=\left\{\frac{1}{x}\right\}=\frac{1}{x}-\left\lfloor\frac{1}{x}\right\rfloor \text { if } x \neq 0, \quad T(0):=0,
$$

associated with the RCF (regular continued fraction) expansion

$$
x=\left[a_{1}, a_{2}, \ldots\right]:=\frac{1}{a_{1}+\frac{1}{a_{2}+\frac{1}{\ddots}}}, \quad a_{i} \in \mathbb{N} .
$$

On such expansions $T$ acts as a one-sided shift

$$
T\left(\left[a_{1}, a_{2}, \ldots\right]\right)=\left[a_{2}, a_{3}, \ldots\right] .
$$

The digits of $x$ are fully recaptured by the $T$-iterates of $x$ as $a_{1}=\left\lfloor\frac{1}{x}\right\rfloor, a_{n+1}=\left\lfloor\frac{1}{T^{n}(x)}\right\rfloor, n \geq 1$. It was discovered by Gauss that the probability measure $\mu_{G}:=\frac{d x}{(1+x) \log 2}$ is $T$-invariant. The endomorphism $T$ is exact in the sense of Rohlin. The measure $\mu_{G}$ is the unique Lebesgue absolutely continuous $T$-invariant probability measure. For a comprehensive presentation of the ergodic properties of $T$ we refer to [21.

Equality (1.1) shows that the periodic points of $T$ are precisely the reduced quadratic irrationals in $[0,1)$, i.e. the numbers with periodic RCF-representation $\omega=\left[\overline{a_{1}, \ldots, a_{n}}\right]$. These are known to coincide with the QIs (quadratic irrationals) $\omega \in[0,1)$ with conjugate $\omega^{*} \in(-\infty,-1]$. An important connection between $\omega$ and $\omega^{*}$ is provided by the Galois formula ([15])

$$
\left[\overline{a_{1}, \ldots, a_{n}}\right]^{*}=-\frac{1}{\left[\overline{a_{n}, \ldots, a_{1}}\right]} .
$$

Date: March 31, 2021.

2020 Mathematics Subject Classification. 37A44 (primary), 11J70, 11N37, 37D40 (secondary).

Key words and phrases. Gauss shift, even continued fraction, backward continued fraction, reduced quadratic irrationals, Pell equation, equidistribution. 
Reduced QIs are naturally ordered by their length

$$
\varrho(\omega):=2 \log \epsilon_{0}(\omega),
$$

where $\epsilon_{0}(\omega)=\epsilon_{\Delta}=\frac{1}{2}\left(u_{0}+v_{0} \sqrt{\Delta}\right)$ is the fundamental solution of the Pell equation $u^{2}-\Delta v^{2}=4$, with $\Delta:=\operatorname{disc}(\omega)$. Geometrically, $\varrho(\omega)$ measures the length of the closed primitive geodesic on the modular surface $\mathscr{M}=\mathrm{SL}(2, \mathbb{Z}) \backslash \mathbb{H}$, which has a lift to $\mathbb{H}$ with endpoints at $\omega^{-1}=$ $\left[\overline{a_{1}, \ldots, a_{n}}\right]^{-1}$ and $\omega^{*}=-\left[\overline{a_{n}, \ldots, a_{1}}\right]$. More concretely, if we set

$$
\Omega(\omega):=\left(\begin{array}{cc}
a_{1} & 1 \\
1 & 0
\end{array}\right) \cdots\left(\begin{array}{cc}
a_{n} & 1 \\
1 & 0
\end{array}\right), \quad \widetilde{\Omega}(\omega):= \begin{cases}\Omega(\omega) & \text { if } n \text { even } \\
\Omega(\omega)^{2} & \text { if } n \text { odd }\end{cases}
$$

and denote by $\mathfrak{r}(\sigma)$ the spectral radius of a $2 \times 2$ matrix $\sigma$, then

$$
\varrho(\omega)=2 \log \mathfrak{r}(\widetilde{\Omega}(\omega)) .
$$

It is well known (see, e.g., [14]) that, in the upper half-plane $\mathbb{H}$, for every $\sigma \in \operatorname{SL}(2, \mathbb{R})$ and $z \in \mathbb{H}$ on the axis of $\sigma$,

$$
d(z, \sigma z)=2 \log \mathfrak{r}(\sigma) .
$$

Employing Mayer's thermodynamic formalism for the Gauss shift $T([23])$ and the Series coding of geodesics on the modular surface $\mathscr{M}([33])$, Pollicott proved ([27], see also Faivre's ensuing work [14]) that the periodic points of $T$ are equidistributed with respect to the Gauss measure $\mu_{G}$, and also that closed geodesics on $\mathscr{M}$ are uniformly distributed when ordered by length. More recently, Kelmer proved a more general result (20]) about closed geodesics with prescribed linking number and the uniform distribution on $[0,1)^{2}$ of the periodic points of the (invertible) natural extension $\widetilde{T}$ of $T$, with respect to the $\widetilde{T}$-invariant probability measure $\widetilde{\mu}_{G}:=\frac{d x d y}{(x y+1)^{2} \log 2}$. These proofs rely on the spectral analysis of the nuclear Perron-Frobenius operator associated to $T$, acting on the disk algebra $A\left(\left\{|z-1| \leq \frac{3}{2}\right\}\right)$, and ultimately on an application of the Wiener-Ikehara tauberian theorem, which does not lead to effective estimates for the error term in the final asymptotic formula. Effective asymptotic results from applications of transfer operators have very recently emerged in the study of the additive cost of moderate growth of reduced QIs ([10]), and respectively of the average of word lengths of closed geodesics on negatively curved surfaces ([8]).

When ordering by discriminant, a powerful number theoretical result of Duke ([13]) shows that the collection of closed geodesics with the same discriminant $\Delta>0$, and hence with the same length $2 \log \epsilon_{\Delta}$, are equidistributed in $\mathscr{M}$.

A more direct number theoretical approach for estimating the number of periodic points of $T$, initiated in [19], was further sharpened by one of the authors ([3]), followed by work of Ustinov [36] (see also the Appendix to [17]). The approach from [3] and [36] relies essentially on applications of the Weil bound for Kloosterman sums. In that setting, the problem was reduced to deriving an asymptotic formula for the number $S(\alpha, \beta ; N)$ of matrices $\left(\begin{array}{cc}p & p^{\prime} \\ q & q^{\prime}\end{array}\right) \in \mathrm{SL}(2, \mathbb{Z})$ subject to the inequalities $\alpha q^{\prime} \geq p^{\prime}>p>0, \beta q^{\prime} \geq q>p$, and $p+q^{\prime} \leq N$, where $\alpha, \beta \in[0,1]$ are fixed and $N \rightarrow \infty$. The following effective estimate was proved in [36]:

$$
\begin{aligned}
S(\alpha, \beta ; N) & =\frac{\log (\alpha \beta+1)}{2 \zeta(2)} N^{2}+O_{\varepsilon}\left(N^{3 / 2+\varepsilon}\right) \\
& =\frac{N^{2}}{2 \zeta(2)} \iint_{[0, \alpha] \times[0, \beta]} \frac{d x d y}{(x y+1)^{2}}+O_{\varepsilon}\left(N^{3 / 2+\varepsilon}\right), \quad \forall \varepsilon>0 .
\end{aligned}
$$


Elementary considerations $([19,3,36])$ then lead to the estimate

$$
\sum_{\substack{\omega \text { reduced Q.I. } \\ \varrho(\omega) \leq R \\ 0 \leq \omega \leq \alpha \\ 0 \leq-1 / \omega^{*} \leq \beta}} 1=\frac{e^{R}}{2 \zeta(2)} \iint_{[0, \alpha] \times[0, \beta]} \frac{d x d y}{(x y+1)^{2}}+O_{\varepsilon}\left(e^{(3 / 4+\varepsilon) R}\right),
$$

showing that the periodic points of the natural extension of $T$ are $\widetilde{\mu}_{G}$-equidistributed.

In Vallée's classification of Euclidean algorithms ([37, 38]), the MSB (most significant bits) class is given special attention. There are six CF (continued fraction) MSB algorithms, denoted by $(\mathrm{G}),(\mathrm{M}),(\mathrm{K}),(\mathrm{E}),(\mathrm{O}),(\mathrm{T})$. In our terminology they are: $(\mathrm{G}) \longleftrightarrow \mathrm{RCF}$ (regular $\mathrm{CF}$ ), $(\mathrm{M})$ $\longleftrightarrow \mathrm{BCF}$ (backward $\mathrm{CF}$ ), $(\mathrm{K}) \longleftrightarrow \mathrm{NICF}$ (nearest integer $\mathrm{CF}$ ), $(\mathrm{E}) \longleftrightarrow \mathrm{ECF}$ (even $\mathrm{CF}),(\mathrm{O})$ $\longleftrightarrow$ OCF (odd CF), $(\mathrm{T}) \longleftrightarrow$ LCF (Lehner CF). The Gauss map corresponding to type (T) is given by $V(x):=\frac{x}{1-x}$ if $x \in\left[0, \frac{1}{2}\right]$ and $V(x):=\frac{1-x}{x}$ if $x \in\left[\frac{1}{2}, 1\right]$. This is the familiar Farey map on $[0,1]$. It was observed in [12] that conjugating by $x \mapsto x+1$ one gets the Gauss map of the Lehner CF on the interval $[1,2]$, which involves only the digits $(1,+1)$ and $(2,-1)$ (see also [24] for a geometric approach). The algorithms (M), (E) and (T) are "slow" and belong to the "Bad Class" (see Section 2.5 of [38]). Incidentally, their associated Gauss shifts have infinite invariant measure, which makes a Perron-Frobenius operator approach as in [2, 17, 20, 27] more challenging.

The analogue of Pollicott's problem for the Farey map has been already thoroughly studied by Heersink ([17], see also [28] for a broader scenery). Building on the approach from [27] and [14], the equidistribution of the periodic points of the Farey map, and also of its natural extension, with respect to their (infinite) invariant measures have been established in [17].

In this paper we investigate the distribution of the periodic points of the Gauss shifts $T_{E}$ in situation (E) and $T_{B}$ in the situation (M). Our results show that, when ordered by appropriate lengths $\varrho_{E}$ and respectively $\varrho_{B}$, these subsets of QIs are equidistributed in an effective manner with respect to the Lebesgue absolutely continuous measure of the corresponding Gauss shift. In fact, Theorems 1 and 4 below show that the periodic points of the natural extensions of these maps are equidistributed with respect to their invariant measure. With the purpose of stating these results, we start with a summary of definitions and properties of the shifts $T_{E}$ and $T_{B}$, and of their periodic points. Here, we prefer to work with ECF and BCF-expansions of numbers in $[1, \infty) \backslash \mathbb{Q}$ rather than $[0,1] \backslash \mathbb{Q}$. Definitions and results can be easily formulated on $[0,1]$ by conjugating by $x \mapsto \frac{1}{x}$. An operator theoretical approach appears to be complicated, a first difficulty being to find an appropriate invariant space of analytic functions under the corresponding Perron-Frobenius operator. Our approach is number theoretical and ultimately relies on the Weil bound for Kloosterman sums.

Every irrational number $u>1$ has a unique ECF-expansion

$$
u=\left[\left(a_{1}, e_{1}\right),\left(a_{2}, e_{2}\right), \ldots\right]:=a_{1}+\frac{e_{1}}{a_{2}+\frac{e_{2}}{a_{3}+\cdots}} \geq 1,
$$

where $a_{i} \in 2 \mathbb{N}$ and $e_{i} \in\{ \pm 1\}$. The corresponding ECF Gauss shift $T_{E}$ acts on $[1, \infty) \backslash \mathbb{Q}$ by $T_{E}\left(\left[\left(a_{1}, e_{1}\right),\left(a_{2}, e_{2}\right), \ldots\right]\right)=\left[\left(a_{2}, e_{2}\right),\left(a_{3}, e_{3}\right), \ldots\right]$. In different notation we have

$$
T_{E}(u)=\left(\begin{array}{cc}
0 & e_{1} \\
1 & -a_{1}
\end{array}\right) u=\frac{e_{1}}{u-a_{1}},
$$

where $a_{1}=a_{1}(u)=2\left\lfloor\frac{u+1}{2}\right\rfloor \in 2 \mathbb{N}$ and $e_{1}=e_{1}(u)=\operatorname{sgn}\left(u-a_{1}(u)\right) \in\{ \pm 1\}$. The infinite measure $\mu_{E}=\left(\int_{-1}^{1}(u+v)^{-2} d v\right) d u=\frac{2 d u}{(u-1)(u+1)}$ is $T_{E}$-invariant. Conjugating by $J(x):=\frac{1}{x}$, one gets the 
customary ECF Gauss map $\bar{T}_{E}:=J^{-1} T_{E} J([22$, 30, 31]), which acts on $[0,1]$ as

$$
\bar{T}_{E}(x)=\left|\frac{1}{x}-2\left[\frac{x+1}{2 x}\right]\right| \text { if } x \neq 0, \quad \bar{T}_{E}(0):=0,
$$

with invariant measure $\nu_{E}=J_{*} \mu_{E}=\frac{2 d x}{(1-x)(1+x)}$. Equivalently, $\bar{T}_{E}$ acts as a shift on ECFexpansions

$$
\bar{T}_{E}\left(\left[\left(a_{1}, e_{1}\right),\left(a_{2}, e_{2}\right),\left(a_{3}, e_{3}\right), \ldots\right]\right)=\left[\left(a_{2}, e_{2}\right),\left(a_{3}, e_{3}\right), \ldots\right] .
$$

The periodic points of $T_{E}$ are exactly the irrationals with periodic ECF-expansion $\omega=$ $\left[\overline{\left(a_{1}, e_{1}\right), \ldots,\left(a_{n}, e_{n}\right)}\right]$. In Section 2 we will show that these are also exactly the elements of the set $\mathscr{R}_{E}$ of QIs $\omega>1$ with $\omega^{*} \in[-1,1]$, which we call E-reduced QIs.

To define the length of $\omega=\left[\overline{\left(a_{1}, e_{1}\right), \ldots,\left(a_{n}, e_{n}\right)}\right] \in \mathscr{R}_{E}$ with $n=\operatorname{per}(\omega)$, we introduce the matrices

$$
\Omega_{E}(\omega):=\left(\begin{array}{cc}
a_{1} & e_{1} \\
1 & 0
\end{array}\right) \cdots\left(\begin{array}{cc}
a_{n} & e_{n} \\
1 & 0
\end{array}\right), \quad \widetilde{\Omega}_{E}(\omega):= \begin{cases}\Omega_{E}(\omega) & \text { if }\left(-e_{1}\right) \cdots\left(-e_{n}\right)=+1 \\
\Omega_{E}(\omega)^{2} & \text { if }\left(-e_{1}\right) \cdots\left(-e_{n}\right)=-1\end{cases}
$$

then set

$$
\varrho_{E}(\omega):=2 \log \mathfrak{r}\left(\widetilde{\Omega}_{E}(\omega)\right), \quad \omega \in \mathscr{R}_{E}
$$

Denote

$$
I_{2}:=\left(\begin{array}{ll}
1 & 0 \\
0 & 1
\end{array}\right), \quad J_{2}:=\left(\begin{array}{ll}
0 & 1 \\
1 & 0
\end{array}\right) .
$$

Consider the Theta groups $\widetilde{\Theta}:=\left\{\sigma \in \mathrm{GL}(2, \mathbb{Z}) \mid \sigma \equiv I_{2}\right.$ or $\left.J_{2}(\bmod 2)\right\}$ and $\Theta:=\widetilde{\Theta} \cap \operatorname{SL}(2, \mathbb{Z})$. In the RCF case, the stabilizer $\{\sigma \in \mathrm{GL}(2, \mathbb{Z}) \mid \sigma \omega=\omega\}$ of a reduced quadratic irrational $\omega$ is used to produce solutions of the Pell equations $u^{2}-\Delta v^{2}= \pm 4$ (see. e.g., [16, 25]). The connection obtained by replacing reduced QIs by $E$-reduced QIs and the group $\operatorname{GL}(2, \mathbb{Z})$ by its subgroup $\widetilde{\Theta}$ will be discussed in Section 3 .

The closed primitive geodesics on the modular surface $\Theta \backslash \mathbb{H}$ correspond exactly (cf. [5]) to those closed geodesics that lift to a geodesic on $\mathbb{H}$ with endpoints

$$
\gamma_{+\infty}=e\left[\overline{\left(a_{1}, e_{1}\right), \ldots,\left(a_{n}, e_{n}\right)}\right]=e \omega \quad \text { and } \quad \gamma_{-\infty}=\gamma_{+\infty}^{*},
$$

for some $e \in\{ \pm 1\}$, and also with

$$
\left(-e_{1}\right) \ldots\left(-e_{n}\right)=1 .
$$

This shows that the $E$-reduced QIs $\omega$ are naturally ordered by $\varrho_{E}(\omega)$, which represents the length of such a geodesic.

The difference between $E$-reduced QIs and ordinary reduced QIs will be illustrated in the Appendix, where we consider the families of $E$-reduced QIs of the form $[\overline{(a,-1)}],\left[\overline{\left(a_{1}, 1\right),\left(a_{2},-1\right)}\right]$, $\left[\overline{\left(a_{1},-1\right),\left(a_{2}, 1\right)}\right]$, and $\left[\overline{\left(a_{1},-1\right),\left(a_{2},-1\right)}\right]$. We compute their discriminants and lengths $\varrho_{E}(\omega)$, and show that only the third family contains regular reduced QIs.

For every $\alpha, \beta_{1}, \beta_{2} \geq 1, N \in \mathbb{N}$, set

$$
r_{E}\left(\alpha, \beta_{1}, \beta_{2} ; R\right):=\sum_{\substack{\omega \in \mathscr{R}_{E}, \varrho_{E}(\omega) \leq R \\ \omega \geq \alpha,-\frac{1}{\beta_{2}} \leq \omega^{*} \leq \frac{1}{\beta_{1}}}} 1 .
$$

We will prove the following results concerning the distribution of periodic points of $T_{E}$ :

Theorem 1. For every $\alpha, \beta_{1}, \beta_{2} \geq 1$ with $\left(\alpha, \beta_{1}\right) \neq(1,1)$,

$$
r_{E}\left(\alpha, \beta_{1}, \beta_{2} ; R\right)=C\left(\alpha, \beta_{1}, \beta_{2}\right) e^{R}+O_{\alpha, \beta_{1}, \varepsilon}\left(e^{(3 / 4+\varepsilon) R}\right), \quad \forall \varepsilon>0,
$$


where

$$
C\left(\alpha, \beta_{1}, \beta_{2}\right)=\frac{1}{\pi^{2}} \log \left(\frac{\alpha \beta_{2}+1}{\alpha \beta_{2}} \cdot \frac{\alpha \beta_{1}}{\alpha \beta_{1}-1}\right)=\frac{1}{\pi^{2}} \iint_{[\alpha, \infty) \times\left[-\frac{1}{\beta_{1}}, \frac{1}{\beta_{2}}\right]} \frac{d u d v}{(u+v)^{2}} .
$$

Taking $\beta_{1}=\beta_{2}=1$, and $\alpha=\beta_{2}=1, \beta_{1}=\infty$ respectively, we find

Corollary 2. For every $\alpha>1$,

Corollary 3. $\sum_{\substack{\omega \in \mathscr{R}_{E}, \omega^{*}<0 \\ \varrho_{E}(\omega) \leq R}} 1=\frac{e^{R} \log 2}{\pi^{2}}+O_{\varepsilon}\left(e^{(3 / 4+\varepsilon) R}\right)$.

Every number $u \in[1, \infty) \backslash \mathbb{Q}$ has a unique BCF-expansion

$$
u=\llbracket a_{1}, a_{2}, a_{3}, \ldots \rrbracket:=a_{1}-\frac{1}{a_{2}-\frac{1}{a_{3}-\frac{1}{\ddots}}} \geq 1, \quad a_{i} \in \mathbb{N}, a_{i} \geq 2 .
$$

The BCF Gauss shift $T_{B}$ acts on $[1, \infty) \backslash \mathbb{Q}$ as $T_{B}\left(\llbracket a_{1}, a_{2}, \ldots \rrbracket\right)=\llbracket a_{2}, a_{3}, \ldots \rrbracket$, or, in different notation,

$$
T_{B}(u)=M\left(a_{1},-1\right)^{-1} u=\left(\begin{array}{cc}
0 & -1 \\
1 & -a_{1}
\end{array}\right) u=\frac{1}{a_{1}-u}=\frac{1}{1-\{u\}},
$$

where $a_{1}=a_{1}(u)=1+\lfloor u\rfloor \geq 2$. The infinite measure $\mu_{B}=\left(\int_{0}^{1}(u-v)^{-2} d v\right) d u=\frac{d u}{u(u-1)}$ is $T_{B}$-invariant. Conjugating by $J_{B}(x):=\frac{1}{1-x}$, one gets the Rényi-Gauss map $\bar{T}_{B}:=J_{B}^{-1} T_{B} J_{B}$, which acts on $[0,1]$ as

$$
\bar{T}_{B}(x)=\left\{\frac{1}{1-x}\right\}, \quad x \neq 1,
$$

with invariant measure $\nu_{B}=J_{B *} \mu_{B}=\frac{d x}{x}([29,1])$. BCF-expansions of rational numbers arise in the study of singularities of complex manifolds ([18]) and in formulae for class numbers ([39]).

When performing elementary computations with backward continued fractions, one can simply take $e_{i}=-1, \forall i \geq 1$ in the ECF-expansions and assume that $a_{i} \geq 2$ are (not necessarily even) integers. In particular, this shows that the sequence $\left(\frac{p_{k}}{q_{k}}\right)$ of convergents of a given number is decreasing. The periodic points of $T_{B}$ are exactly the irrationals with periodic BCF-expansion $\omega=\llbracket \overline{a_{1}, \ldots, a_{n}} \rrbracket$. These are shown to also coincide with the elements of the set $\mathscr{R}_{B}$ of QIs $\omega>1$ with $\omega^{*} \in[0,1]$, which we call $B$-reduced QIs. Consider the matrix

$$
\Omega_{B}(\omega):=\left(\begin{array}{cc}
a_{1} & -1 \\
1 & 0
\end{array}\right) \cdots\left(\begin{array}{cc}
a_{n} & -1 \\
1 & 0
\end{array}\right), \quad \text { where } n=\operatorname{per}(\omega) .
$$

Notice that $\operatorname{det}\left(\Omega_{B}(\omega)\right)=+1$, so $\widetilde{\Omega}_{B}(\omega)=\Omega_{B}(\omega)$ for all $\omega \in \mathscr{R}_{B}$. Define

$$
\varrho_{B}(\omega):=2 \log \mathfrak{r}\left(\Omega_{B}(\omega)\right), \quad \omega \in \mathscr{R}_{B} .
$$

Define also

$$
r_{B}(\alpha, \beta ; R):=\sum_{\substack{\omega \in \mathscr{R}_{B}, \varrho_{B}(\omega) \leq R \\ \omega \geq \alpha, 0<\omega^{*} \leq \frac{1}{\beta}}} 1 .
$$

We will prove the following results concerning the distribution of periodic points of $T_{B}$ : 
Theorem 4. For every $\alpha, \beta \geq 1$ with $(\alpha, \beta) \neq(1,1)$,

$$
r_{B}(\alpha, \beta ; R)=\frac{e^{R}}{2 \zeta(2)} \iint_{[\alpha, \infty) \times\left[0, \frac{1}{\beta}\right]} \frac{d u d v}{(u-v)^{2}}+O_{\alpha, \beta_{1}, \varepsilon}\left(e^{(3 / 4+\varepsilon) R}\right), \quad \forall \varepsilon>0 .
$$

Taking $\beta=1$ we find

Corollary 5. For every $\alpha>1$,

$$
\begin{aligned}
\sum_{\substack{\omega \in \mathscr{R}_{B}, \omega \geq \alpha \\
\varrho_{B}(\omega) \leq R}} 1 & =\frac{e^{R}}{2 \zeta(2)} \log \left(\frac{\alpha}{\alpha-1}\right)+O_{\varepsilon}\left(e^{(3 / 4+\varepsilon) R}\right) \\
& =\frac{e^{R}}{2 \zeta(2)} \int_{[\alpha, \infty)} \frac{d u}{u(u-1)}+O_{\alpha, \varepsilon}\left(e^{(3 / 4+\varepsilon) R}\right) .
\end{aligned}
$$

Remark 6. A shorter proof can be achieved without the analytic number theoretical estimates (5.7) and (5.8), but with the price of getting an error term $O_{\varepsilon}\left(e^{(7 / 8+\varepsilon) R}\right)$ instead of $O_{\varepsilon}\left(e^{(3 / 4+\varepsilon) R}\right)$ in Theorems 1 and 4 .

We expect our approach to also work in the remaining situations of (good) MSB Euclidean algorithms of type $(\mathrm{K})$ and $(\mathrm{O})$. We are planning to study this in further work.

\section{Even Continued Fractions}

In this section we investigate various algebraic properties of ECF expansions. In Subsection 2.1 we revisit the main features of the ECF Gauss shift, and in Subsection 2.2 we focus on QIs. Among other things, we give a short algebraic proof of the ECF analogue of the Galois formula (1.2) (previously proved in different ways in [22] and [5]), then prove that ECF-periodic QIs coincide exactly with QIs $\omega>1$ having $\omega^{*} \in(-1,1)$ (previously proved geometrically in [5]). Finally, inspired by the approach pursued in the RCF situation in [19, 3, 36], we describe some bijections between subsets of $\mathscr{R}_{E}$ and subsets of $\operatorname{SL}(2, \mathbb{Z})$ that will play an essential role in the proof of Theorem 1 .

2.1. ECF expansions and the associated Gauss shift. The ECF-convergents $\frac{p_{k}}{q_{k}}$ of an irrational number $u$ as in (1.6) are given by

$$
\begin{aligned}
& p_{0}=p_{0}(u):=1, \quad p_{1}=p_{1}(u):=a_{1}, \quad p_{k}=p_{k}(u):=a_{k} p_{k-1}+e_{k-1} p_{k-2}, \\
& q_{0}=q_{0}(u):=0, \quad q_{1}=q_{1}(u):=1, \quad q_{k}=q_{k}(u):=a_{k} q_{k-1}+e_{k-1} q_{k-2} .
\end{aligned}
$$

These relations show

$$
q_{k} \text { even } \Longleftrightarrow k \text { even } \Longleftrightarrow p_{k-1} \text { even. }
$$

The matrix $M(a, e):=\left(\begin{array}{ll}a & e \\ 1 & 0\end{array}\right)$ acts on $u$ by

$$
M(a, e) u=a+\frac{e}{u}=[(a, e), u] .
$$

The Gauss map $T_{E}$ acts on $u=\left[\left(a_{1}, e_{1}\right),\left(a_{2}, e_{2}\right), \ldots\right]$ by

$$
T_{E}(u)=M\left(a_{1}, e_{1}\right)^{-1} u=\left(\begin{array}{cc}
0 & e_{1} \\
1 & -a_{1}
\end{array}\right) u=\frac{e_{1}}{u-a_{1}} .
$$

The natural extension of the endomorphism $T_{E}$ is the automorphism of $[1, \infty) \times[-1,1]$ given by

$$
\widetilde{T}_{E}(u, v)=\left(T_{E}(u), \frac{e_{1}}{v+a_{1}}\right)=\left(\frac{e_{1}}{u-a_{1}}, \frac{e_{1}}{v+a_{1}}\right) .
$$



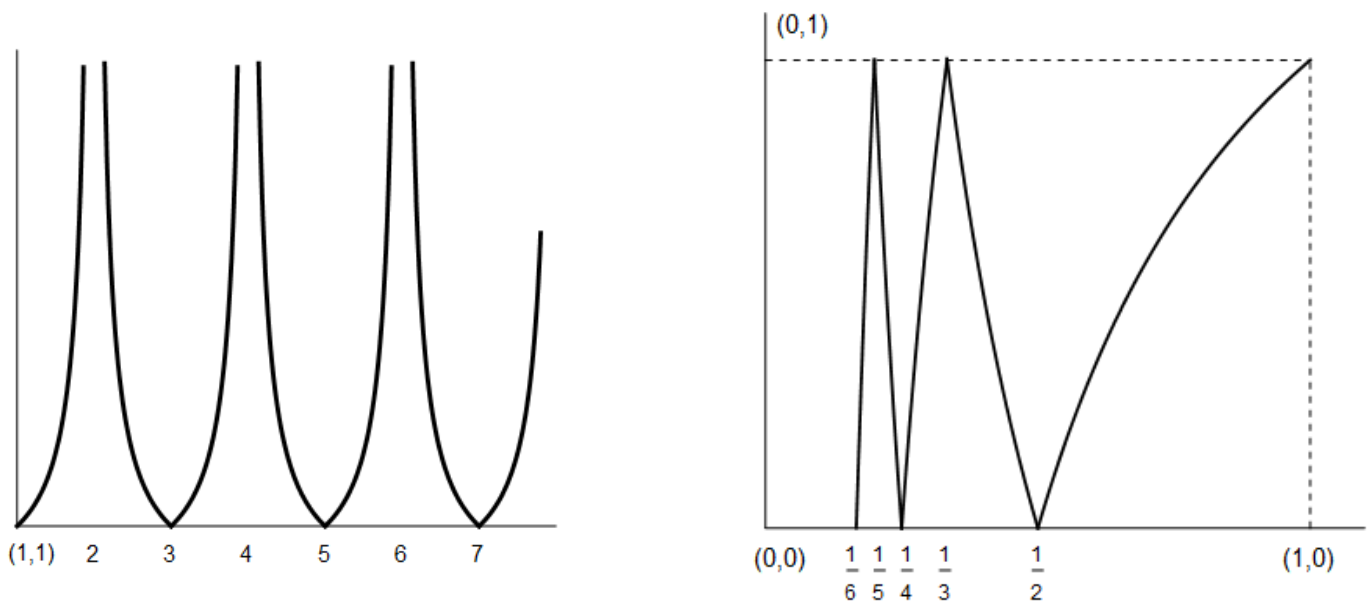

Figure 1. The graphs of the maps $T_{E}$ and $\bar{T}_{E}$

The map $\widetilde{T}_{E}$ acts on the digits in the following way:

$\widetilde{T}_{E}\left(\left[w_{1}, w_{2}, \ldots\right],\left\langle\left\langle w_{0}, w_{-1}, \ldots\right\rangle\right\rangle\right)=\left(\left[w_{2}, w_{3}, \ldots\right],\left\langle\left\langle w_{1}, w_{0}, \ldots\right\rangle\right\rangle\right), \quad w_{i}=\left(a_{i}, e_{i}\right) \in 2 \mathbb{N} \times\{ \pm 1\}$,

where

$$
\left\langle\left\langle\left(b_{1}, f_{1}\right),\left(b_{2}, f_{2}\right), \ldots\right\rangle\right\rangle:=\frac{f_{1}}{b_{1}+\frac{f_{2}}{b_{2}+\ddots}}, \quad b_{j} \in 2 \mathbb{N}, f_{j} \in\{ \pm 1\}
$$

denotes the dual ECF-expansion of irrationals in $[-1,1]$.

From general ergodic theoretical considerations ([5]), the infinite measure $(u+v)^{-2} d u d v$ is $\widetilde{T}_{E}$-invariant, while $d \mu_{E}=\left(\int_{-1}^{1}(u+v)^{-2} d v\right) d u=\frac{2 d u}{(u-1)(u+1)}$ is $T_{E}$-invariant.

Conjugating through $J(x):=\frac{1}{x}$, one gets the customary ECF Gauss map $\bar{T}_{E}:=J^{-1} T_{E} J$ $([30,31,22,9])$, which acts on $[0,1]$ as in formula (1.7), with invariant measure $\nu_{E}=J_{*} \mu_{E}=$ $\frac{2 d x}{(1-x)(1+x)}$. The endomorphism $\bar{T}_{E}$ is exact in Rohlin's sense (this can be proved exactly as in the ECF situation using the approach from [32]), and $\nu_{E}$ is the unique $\sigma$-finite Lebesgue absolutely

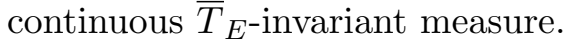

We introduce the matrices

$$
\Omega_{k}(u):=M\left(a_{1}, e_{1}\right) \cdots M\left(a_{k}, e_{k}\right)=\left(\begin{array}{cc}
p_{k} & p_{k-1} e_{k} \\
q_{k} & q_{k-1} e_{k}
\end{array}\right),
$$

with determinant

$$
\operatorname{det}\left(\Omega_{k}(u)\right)=\left(p_{k} q_{k-1}-p_{k-1} q_{k}\right) e_{k}=\left(-e_{1}\right) \cdots\left(-e_{k}\right),
$$

and inverse

$$
\Omega_{k}(u)^{-1}=\left(-e_{1}\right) \cdots\left(-e_{k}\right)\left(\begin{array}{cc}
q_{k-1} e_{k} & -p_{k-1} e_{k} \\
-q_{k} & p_{k}
\end{array}\right) .
$$

The iterates of $T_{E}$ can be conveniently described as

$$
T_{E}^{k}(u)=\left[\left(a_{k+1}, e_{k+1}\right),\left(a_{k+2}, e_{k+2}\right), \ldots\right]=\Omega_{k}(u)^{-1} u=\left(-e_{k}\right) \frac{p_{k-1}-q_{k-1} u}{p_{k}-q_{k} u},
$$

leading to

$$
T_{E}(u) T_{E}^{2}(u) \cdots T_{E}^{k}(u)=\frac{\left(-e_{1}\right) \cdots\left(-e_{k}\right)}{p_{k}-q_{k} u}, \quad \forall k \geq 1,
$$


and to

Formula (2.3) also gives

$$
u=\frac{e_{k} p_{k-1}+T_{E}^{k}(u) p_{k}}{e_{k} q_{k-1}+T_{E}^{k}(u) q_{k}} .
$$

$$
q_{k-1} e_{k}+T_{E}^{k}(u) q_{k}=e_{k}\left(q_{k-1}+\frac{p_{k-1} q_{k}-u q_{k-1} q_{k}}{u q_{k}-p_{k}}\right)=\frac{\left(-e_{1}\right) \cdots\left(-e_{k}\right)}{p_{k}-u q_{k}},
$$

leading to

$$
u-\frac{p_{k}}{q_{k}}=-\frac{\left(-e_{1}\right) \cdots\left(-e_{k}\right)}{q_{k-1} e_{k}+T_{E}^{k}(u) q_{k}} .
$$

As $T_{E}^{k}(u) q_{k}+q_{k-1} e_{k}>q_{k}-q_{k-1} \geq 1$, this shows

$$
\operatorname{sgn}\left(u-\frac{p_{k}}{q_{k}}\right)=-\left(-e_{1}\right) \cdots\left(-e_{k}\right), \quad \forall k \geq 1 .
$$

2.2. E-reduced quadratic irrationals. In the sequel we extend some properties of QIs from the RCF setting (as considered in [19, 25]) to the ECF setting.

Remark 7. The following facts concerning the discriminant and the conjugate of some QIs $u$ hold for every $\sigma \in \mathrm{GL}(2, \mathbb{Z})$ :

(A) $\operatorname{disc}(\sigma u)=\operatorname{disc}(u)$.

(B) $(\sigma u)^{*}=\sigma u^{*}$.

Lemma 8. Let $\omega$ be a $Q I$, and let $\sigma=\left(\begin{array}{ll}a & b \\ c & d\end{array}\right) \in \mathrm{GL}(2, \mathbb{Z})$ such that $\sigma \omega=\omega$.

(i) The eigenvalues of $\sigma$ are $\lambda_{1}=c \omega+d, \lambda_{2}=\frac{\delta}{c \omega+d}$, where $\delta:=a d-b c \in\{ \pm 1\}$.

(ii) The eigenvalues of $\sigma^{-1}=\delta\left(\begin{array}{cc}d & -b \\ -c & a\end{array}\right)$ are $\mu_{1}=\delta(-c \omega+a)=\frac{1}{\lambda_{1}}$ and $\mu_{2}=\frac{1}{-c \omega+a}=\frac{1}{\lambda_{2}}$.

(iii) $(c \omega+d)^{*}=c \omega^{*}+d$.

Proof. (i) Clearly $\lambda_{1} \lambda_{2}=\operatorname{det}(\sigma)$, thus one only has to check that $c \omega+d+\frac{\delta}{c \omega+d}=a+d$. This is immediately seen to be equivalent to $c \omega(c \omega+d)=c(a \omega+b)$, or equivalently to $\sigma \omega=\omega$.

(ii) From the first part, $\operatorname{Eig}\left(\sigma^{-1}\right)=\left\{\frac{1}{\lambda_{1}}, \frac{1}{\lambda_{2}}\right\}$. More precisely, by direct calculation $\mu_{1}=\frac{1}{\lambda_{1}}$ is equivalent to $\sigma \omega=\omega$.

(iii) The number $\eta:=c \omega+d$ satisfies $\eta^{2}-(a+d) \eta+a d-b c=0$, so $\eta+\eta^{*}=a+d$. On the other hand the equality $c \omega^{*}+(d-a) \omega^{*}-b=0$ provides $\omega+\omega^{*}=\frac{a-d}{c}$. This leads to $a-c \omega=d+c \omega^{*}$ and so $\eta^{*}=a+d-\eta=c \omega^{*}+d$.

Denote by $\mathfrak{N}(u)$ and respectively $\mathfrak{t r}(u)$ the norm and trace of the QI $u$ in the associated quadratic field.

Lemma 9. Let $G$ be a subgroup of $\mathrm{GL}(2, \mathbb{Z})$. For every $Q I \omega$, the map

$$
\Lambda_{\omega}: G_{\omega} \longrightarrow \mathbb{R}, \quad \Lambda_{\omega}\left(\begin{array}{ll}
a & b \\
c & d
\end{array}\right):=c \omega+d,
$$

defines an injective group homomorphism on $G_{\omega}:=\{\sigma \in G \mid \sigma \omega=\omega\}$ with $\mathfrak{N} \circ \Lambda_{\omega}=$ det and $\mathfrak{t r} \circ \Lambda_{\omega}=\operatorname{Tr}$.

Proof. Let $\sigma=\left(\begin{array}{ll}a & b \\ c & d\end{array}\right) \in G$ and $\sigma^{\prime}=\left(\begin{array}{ll}a^{\prime} & b^{\prime} \\ c^{\prime} & d^{\prime}\end{array}\right) \in G_{\omega}$. By definition, $\Lambda_{\omega}$ maps the product matrix $\sigma \sigma^{\prime}$ into $\left(a^{\prime} c+c^{\prime} d\right) \omega+b^{\prime} c+d^{\prime} d$. This coincides with $(c \omega+d)\left(c^{\prime} \omega+d^{\prime}\right)=\Lambda_{\omega}(\sigma) \Lambda_{\omega}\left(\sigma^{\prime}\right)$ as a result of $\frac{a^{\prime} \omega+b^{\prime}}{c^{\prime} \omega+d^{\prime}}=\omega$.

Let $\sigma \in \operatorname{Ker}\left(\Lambda_{\omega}\right)$, so $c \omega+d=1$. The irrationality of $\omega$ yields $c=0$ and $d=1$. Then $\omega=a \omega+b$, hence $a=1$ and $b=0$.

The equalities $\mathfrak{N} \circ \Lambda_{\omega}=\operatorname{det}$ and $\mathfrak{t r} \circ \Lambda_{\omega}=\operatorname{Tr}$ follow from $(c \omega+d)^{*}=c \omega^{*}+d$. 
In the sequel we will consider an ECF-periodic QI $\omega=\left[\overline{\left(a_{1}, e_{1}\right), \ldots,\left(a_{n}, e_{n}\right)}\right]>1$ with $n=\operatorname{per}(\omega)$. Set

$$
\begin{aligned}
\delta_{n}=\delta_{n}(\omega) & :=\left(-e_{1}\right) \cdots\left(-e_{n}\right), \quad \ell=\operatorname{eper}(\omega):= \begin{cases}n & \text { if } \delta_{n}=+1 \\
2 n & \text { if } \delta_{n}=-1,\end{cases} \\
\Omega_{E}(\omega) & :=\Omega_{n}(\omega)=M\left(a_{1}, e_{1}\right) \cdots M\left(a_{n}, e_{n}\right)=\left(\begin{array}{ll}
p_{n} & p_{n-1} e_{n} \\
q_{n} & q_{n-1} e_{n}
\end{array}\right), \\
\widetilde{\Omega}_{E}(\omega) & := \begin{cases}\Omega_{E}(\omega) & \text { if } \delta_{n}=+1 \\
\Omega_{E}(\omega)^{2} & \text { if } \delta_{n}=-1 .\end{cases}
\end{aligned}
$$

Employing (2.4), Lemma 8, $T_{E}^{n}(\omega)=\omega$, and $\delta_{\ell}=+1$, we infer that the spectral radius of $\widetilde{\Omega}_{E}(\omega)$ is

$$
\mathfrak{r}\left(\widetilde{\Omega}_{E}(\omega)\right)=\omega T_{E}(\omega) \cdots T_{E}^{\mathrm{eper}(\omega)-1}(\omega)=q_{\ell} \omega+q_{\ell-1} e_{\ell}=\frac{1}{p_{\ell}-q_{\ell} \omega}>1 .
$$

As it will be seen later, formula (2.4) provides an ECF-analogue of Smith's formula ([34]).

The following ECF-analogue of the Galois formula $[1.2)$ is known $([5,22])$. Here, we give yet another proof, using our setting and the dual ECF-expansion on $[-1,1]([30,31])$.

Lemma 10. The conjugate $\omega^{*}$ of $\omega=\left[\overline{\left(a_{1}, e_{1}\right), \ldots,\left(a_{n}, e_{n}\right)}\right]>1$ is given by

$$
-\omega^{*}=\left\langle\left\langle\overline{\left(a_{n}, e_{n}\right), \ldots,\left(a_{1}, e_{1}\right)}\right\rangle \in(-1,1) .\right.
$$

Proof. The equality $\Omega_{E}(\omega) \omega=\omega$ and formulas 2.2 and 2.3 for $\Omega_{E}(\omega)^{-1}=\Omega_{n}(\omega)^{-1}$ show

$$
\left(\begin{array}{cc}
q_{n-1} e_{n} & -p_{n-1} e_{n} \\
-q_{n} & p_{n}
\end{array}\right) \omega=\left(\begin{array}{cc}
-q_{n-1} e_{n} & p_{n-1} e_{n} \\
q_{n} & -p_{n}
\end{array}\right) \omega=\omega
$$

The conjugate $\omega^{*}$ must also be fixed by the matrix $\left(\begin{array}{cc}-q_{n-1} e_{n} & p_{n-1} e_{n} \\ q_{n} & -p_{n}\end{array}\right)$, and so

$$
\left(\begin{array}{cc}
q_{n-1} e_{n} & p_{n-1} e_{n} \\
q_{n} & p_{n}
\end{array}\right)\left(-\omega^{*}\right)=-\omega^{*} .
$$

Denote $\widetilde{\omega}:=\left\langle\left\langle\overline{\left(a_{n}, e_{n}\right), \ldots,\left(a_{1}, e_{1}\right)}\right\rangle\right.$. Since $\left(\begin{array}{ll}0 & e_{1} \\ 1 & a_{1}\end{array}\right) x=\frac{e_{1}}{a_{1}+x}$, we have $\widetilde{\omega}=\left(\begin{array}{ll}0 & e_{n} \\ 1 & a_{n}\end{array}\right) \cdots\left(\begin{array}{ll}0 & e_{1} \\ 1 & a_{1}\end{array}\right) \widetilde{\omega}$. Since $\left(\begin{array}{ll}0 & e_{1} \\ 1 & a_{1}\end{array}\right)=\left(\begin{array}{cc}q_{0} e_{1} & p_{0} e_{1} \\ q_{1} & p_{1}\end{array}\right)$ and $\left(\begin{array}{ll}0 & e_{k} \\ 1 & a_{k}\end{array}\right)\left(\begin{array}{cc}q_{k-2} e_{k-1} & p_{k-2} e_{k-1} \\ q_{k-1} & p_{k-1}\end{array}\right)=\left(\begin{array}{cc}q_{k-1} e_{k} & p_{k-1} e_{k} \\ q_{k} & p_{k}\end{array}\right)$, we gather

$$
\left(\begin{array}{cc}
q_{n-1} e_{n} & p_{n-1} e_{n} \\
q_{n} & p_{n}
\end{array}\right) \widetilde{\omega}=\widetilde{\omega} .
$$

Suppose that $\widetilde{\omega} \neq-\omega^{*}$. Then, from 2.7, 2.8 and 2.6 we infer $-\omega^{*} \widetilde{\omega}=-\frac{p_{n-1} e_{n}}{q_{n}}=\omega \omega^{*}$. This leads to a contradiction because $\omega>1>|\widetilde{\omega}|$, and so $-\omega^{*}=\widetilde{\omega}$.

Definition 11. Given $\Delta \in \mathbb{N}, \Delta$ not a square, denote

$$
\mathscr{X}(\Delta):=\{\omega Q I \mid \operatorname{disc}(\omega)=\Delta\} .
$$

The $Q I \omega>1$ is called E-reduced if $-1<\omega^{*}<1$. We denote

$$
\mathscr{R}_{E}(\Delta):=\{\omega \in \mathscr{X}(\Delta) \mid \omega \text { E-reduced }\} \quad \text { and } \quad \mathscr{R}_{E}:=\bigcup_{\Delta>0} \mathscr{R}_{E}(\Delta) .
$$

Lemma 12. The set $\mathscr{R}_{E}(\Delta)$ is finite. 
Proof. Finiteness of the set $\left\{\omega \in \mathscr{X}(\Delta) \mid \omega>1,0>\omega^{*}>-1\right\}$ is well known, so it suffices to show that there are only finitely many numbers $\omega \in \mathscr{X}(\Delta)$ such that $\omega>1>\omega^{*}>0$. Let $A X^{2}+B X+C$ be the minimal polynomial of such $\omega$, with $A>0$ and $(A, B, C)=1$, $\Delta=B^{2}-4 A C$. From $\omega \omega^{*}=\frac{C}{A}$ and $\omega+\omega^{*}=-\frac{B}{A}$ we infer $C>0$, respectively $B<-A<0$. The inequalities $\omega=\frac{-B+\sqrt{\Delta}}{2 A}>1>\omega^{*}=\frac{-B-\sqrt{\Delta}}{2 A}>0$ yield

$$
-\sqrt{\Delta}<2 A+B<\sqrt{\Delta} .
$$

After squaring and dividing by $A$, this leads to $0<A+C<-B$. Squaring again we get $(A-C)^{2}<\Delta$, or

$$
-\sqrt{\Delta}<A-C<\sqrt{\Delta} .
$$

Upon (2.9) and (2.10), we can write $2 A+B=\alpha, A-C=\beta$ with $|\alpha|,|\beta|<\sqrt{\Delta}$, and so $(\alpha-2 A)^{2}=B^{2}=\Delta+4 A(A-\beta)$, or equivalently $A(\beta-\alpha)=\Delta-\alpha^{2}$. Since $\Delta$ is not a square (so $\beta \neq \alpha$ ), this gives $0<A=\frac{\left|\Delta-\alpha^{2}\right|}{|\beta-\alpha|} \leq \Delta$, and 2.9) and 2.10) show that $B$ and $C$ can also take only finitely many values.

Lemma 13. If $\omega \in \mathscr{R}_{E}(\Delta)$, then $\beta:=T_{E}(\omega) \in \mathscr{R}_{E}(\Delta)$.

Proof. Let $\omega=\left[\left(a_{1}, e_{1}\right),\left(a_{2}, e_{2}\right), \ldots\right] \in \mathscr{R}_{E}(\Delta)$. Then $\beta=\left(\begin{array}{cc}0 & 1 \\ e_{1} & -e_{1} a_{1}\end{array}\right) \omega$ and $\operatorname{disc}(\beta)=\operatorname{disc}(\omega)=$ $\Delta$. From $\omega=a_{1}+\frac{e_{1}}{\beta}$ it follows that $a_{1}+\frac{e_{1}}{\beta^{*}}=\omega^{*} \in(-1,1)$, showing $\frac{e_{1}}{\beta^{*}}<1-a_{1}$. Hence $\frac{1}{\left|\beta^{*}\right|}>a_{1}-1 \geq 1$, showing $\beta^{*} \in(-1,1)$.

Proposition 14. For every $\omega \in \mathscr{X}(\Delta)$ the following are equivalent:

(i) $\omega \in \mathscr{R}_{E}(\Delta)$.

(ii) $\operatorname{ECF}(\omega)$ is periodic.

(iii) $\omega$ is a periodic point of the map $T_{E}$.

Proof. (ii) $\Longrightarrow$ (i) follows from Lemma 10. If $\operatorname{ECF}(\omega)$ is periodic, i.e. $\omega=\left[\overline{\left(a_{1}, e_{1}\right), \ldots,\left(a_{n}, e_{n}\right)}\right]>$ 1 , then $\omega^{*}=-\left\langle\left\langle\overline{\left(a_{n}, e_{n}\right), \ldots,\left(a_{1}, e_{1}\right)}\right\rangle\right\rangle \in(-1,1)$, hence $\omega \in \mathscr{R}_{E}(\Delta)$.

(i) $\Longrightarrow$ (ii) Let $\omega=\left[\left(a_{1}, e_{1}\right),\left(a_{2}, e_{2}\right), \ldots\right] \in \mathscr{R}_{E}(\Delta)$. Since the set $\mathscr{R}_{E}(\Delta)$ is finite, there exist $r \geq 0$ and $n \geq 1$ such that $T_{E}^{r}(\omega)=T_{E}^{r+n}(\omega)$. The uniqueness of ECF-expansions then shows $\left(a_{s}, e_{s}\right)=\left(a_{s+n}, e_{s+n}\right), \forall s \geq r+1$.

It remains to show that if $k \geq 1$ and $T_{E}^{k}(\omega)=T_{E}^{k+n}(\omega)$, then $T_{E}^{k-1}(\omega)=T_{E}^{k+n-1}(\omega)$. Set $\omega_{i}:=T_{E}^{i}(\omega)$. By Lemma 13, $\omega_{k-1}=\left[\left(a_{k}, e_{k}\right), \omega_{k}\right]=a_{k}+\frac{e_{k}}{\omega_{k}} \in \mathscr{R}_{E}(\Delta)$, whence $\omega_{k-1}^{*}=a_{k}+\frac{e_{k}}{\omega_{k}^{*}} \in$ $(-1,1)$. Similarly, from $\omega_{k+n-1}=\left[\left(a_{k+n}, e_{k+n}\right), \omega_{k+n}\right]=a_{k+n}+\frac{e_{k+n}}{\omega_{k+n}}$ and $\omega_{k+n} \in \mathscr{R}_{E}(\Delta)$ it follows that $\omega_{k+n-1}^{*}=a_{k+n}+\frac{e_{k+n}}{\omega_{k+n}^{*}} \in(-1,1)$. But $\omega_{k}=\omega_{k+n}$ entails $\omega_{k}^{*}=\omega_{k+n}^{*}=: \beta \in(-1,1)$, and thus $-1<a_{k}+\frac{e_{k}}{\beta}<1$ and $-1<a_{k+n}+\frac{e_{k+n}}{\beta}<1$, or equivalently

$$
e_{k} a_{k}, e_{k+n} a_{k+n} \in\left(-1-\frac{1}{\beta}, 1-\frac{1}{\beta}\right) \text {. }
$$

Since $a_{k}$ and $a_{k+n}$ are even, this gives $\left(a_{k}, e_{k}\right)=\left(a_{k+n}, e_{k+n}\right)$. Letting $k$ decrease by one (unless $k=0$ ), one finds $\omega=T_{E}^{n}(\omega)$, showing that $\operatorname{ECF}(\omega)$ is periodic.

(ii) $\Longleftrightarrow$ (iii) follows from the first equality in $(2.3)$.

The ECF version of Lagrange's theorem is well known. It also holds for larger classes of continued fractions, including backward continued fractions.

Proposition $15([22,26,11])$. For every $u \in[1, \infty) \backslash \mathbb{Q}$ the following are equivalent:

(i) $u$ is a $Q I$. 
(ii) $E C F(u)$ is eventually periodic, i.e.

$$
u=\left[\left(a_{1}, e_{1}\right), \ldots,\left(a_{r}, e_{r}\right), \overline{\left(a_{r+1}, e_{r+1}\right), \ldots,\left(a_{r+n}, e_{r+n}\right)}\right] .
$$

We consider the Theta groups $\widetilde{\Theta}$ and $\Theta$ defined in the introduction.

Lemma 16. For every $u \in \mathscr{X}(\Delta), \widetilde{\Theta} u \cap \mathscr{R}_{E}(\Delta) \neq \emptyset$.

Proof. The previous proposition allows us to take $u$ as in 2.11. Setting

$$
\sigma:=\left(\begin{array}{cc}
0 & 1 \\
e_{r} & -e_{r} a_{r}
\end{array}\right) \cdots\left(\begin{array}{cc}
0 & 1 \\
e_{1} & -e_{1} a_{1}
\end{array}\right) \in \widetilde{\Theta},
$$

we have

$$
\sigma u=\left[\overline{\left(a_{r+1}, e_{r+1}\right), \ldots,\left(a_{r+n}, e_{r+m}\right)}\right] \in \widetilde{\Theta} u \cap \mathscr{R}_{E}(\Delta) .
$$

2.3. Some bijections between subsets of $\mathscr{R}_{E}$ and subsets of $\operatorname{SL}(2, \mathbb{Z})$. Consider the collections of matrices

$$
\begin{aligned}
& \mathscr{P}:=\left\{M\left(a_{1}, e_{1}\right) \cdots M\left(a_{n}, e_{n}\right) \mid n \geq 1, a_{i} \in 2 \mathbb{N}, e_{i} \in\{ \pm 1\}\right\} \quad \text { and } \\
& \mathscr{S}:=\left\{\sigma=\left(\begin{array}{cc}
p^{\prime} & p e \\
q^{\prime} & q e
\end{array}\right) \in \mathrm{GL}(2, \mathbb{Z}) \mid \begin{array}{c}
\sigma \equiv I_{2} \text { or } J_{2} \quad(\bmod 2), e \in\{ \pm 1\} \\
p^{\prime}>p>q>0, p^{\prime}>q^{\prime}>q
\end{array}\right\} .
\end{aligned}
$$

Proposition 17. $\mathscr{P}=\mathscr{S}$.

Proof. $(\subseteq)$ The matrix $M\left(a_{1}, e_{1}\right) \cdots M\left(a_{n}, e_{n}\right)=\left(\begin{array}{cc}p_{n} & p_{n-1} e_{n} \\ q_{n} & q_{n-1} e_{n}\end{array}\right)$ is $\equiv I_{2}$ or $J_{2}(\bmod 2)$ as a product of matrices $M\left(a_{i}, e_{i}\right) \equiv J_{2}(\bmod 2)$. We also have $q_{1}=1>q_{0}=0$, and by induction $q_{n}=$ $a_{n} q_{n-1}+e_{n-1} q_{n-2} \geq 2 q_{n-1}-q_{n-2}>q_{n-1}$. Similarly, $p_{n}>p_{n-1}$ as $p_{1}=a_{1}>p_{0}=1$ and $p_{n}-q_{n} \geq p_{n-1}-q_{n-1} \geq p_{1}-q_{1}=a_{1}-1 \geq p_{0}-q_{0}=1$, showing $M\left(a_{1}, e_{1}\right) \cdots M\left(a_{n}, e_{n}\right) \in \mathscr{S}$.

$(\supseteq)$ Let $\sigma:=\left(\begin{array}{c}p^{\prime} p e \\ q^{\prime} q e\end{array}\right) \in \mathscr{S}$. Consider $a:=2\left\lfloor\frac{q^{\prime}}{2 q}+\frac{1}{2}\right\rfloor \geq 2$. The inverse of $M(a, e)$ is $M(a, e)^{-1}=(-e)\left(\begin{array}{cc}0 & -e \\ -1 & a\end{array}\right)$ and

$$
\sigma_{0}:=\sigma M(a, e)^{-1}=(-e)\left(\begin{array}{cc}
p^{\prime} & p e \\
q^{\prime} & q e
\end{array}\right)\left(\begin{array}{cc}
0 & -e \\
-1 & a
\end{array}\right)=\left(\begin{array}{cc}
p & p^{\prime}-a p \\
q & q^{\prime}-a q
\end{array}\right) \in \mathrm{GL}(2, \mathbb{Z})
$$

with $\sigma_{0} \equiv I_{2}$ or $J_{2}(\bmod 2)$. Upon $\frac{q^{\prime}}{2 q}-\frac{1}{2}<\frac{a}{2} \leq \frac{q^{\prime}}{2 q}+\frac{1}{2}$ we have $-q \leq q^{\prime}-a q<q$. Since $\left(q^{\prime}, q\right)=1$, if $q^{\prime}-a q=-q$ then $q=1$, which in turn implies $q^{\prime} \equiv q \bmod 2$, contradiction. Hence $q^{\prime}-a q=f q_{0}$ with $f=\operatorname{sgn}\left(q^{\prime}-a q\right) \in\{ \pm 1\}$ and $0<q_{0}<q$.

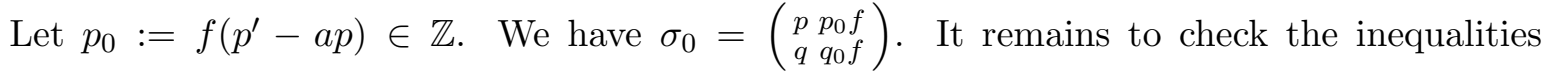
$p>p_{0}>0$ and $p_{0}>q_{0}$, and to investigate what happens when $q_{0}=1$.

Upon $\operatorname{det}\left(\sigma_{0}\right)=f\left(q p_{0}-p q_{0}\right) \in\{ \pm 1\}$ we have $p_{0}=\frac{p q_{0} \pm 1}{q}=\frac{p}{q} q_{0} \pm \frac{1}{q}>q_{0}-\frac{1}{q} \geq q_{0}-1$, showing $p_{0} \geq q_{0} \geq 1$. Since $\left(p_{0}, q_{0}\right)=1$, then either $p_{0}>q_{0}$ or $q_{0}=1$. Since $q-q_{0} \geq 1$ and $p \geq 2$ as $p>q>q_{0}$, we have $p\left(q-q_{0}\right)>1$, showing $p>\frac{p q_{0}+1}{q} \geq p_{0}$.

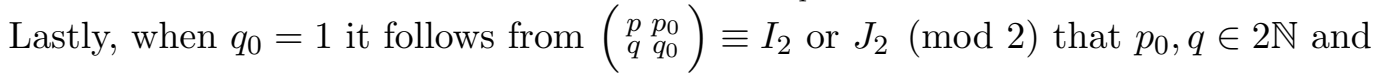

$$
\sigma_{0}=\left(\begin{array}{cc}
p & p_{0} e \\
q & e
\end{array}\right)=\left(\begin{array}{cc}
p_{0} & f \\
1 & 0
\end{array}\right)\left(\begin{array}{ll}
q & e \\
1 & 0
\end{array}\right)=M\left(p_{0}, f\right) M(q, e),
$$

where $f \in\{ \pm 1\}$ is given by $f=p-p_{0} q=p q_{0}-p_{0} q$. 
Remark 18. The defining relations for $q_{n}$ and $p_{n}$ lead to $\frac{q_{2}}{q_{1}}=a_{2}, \frac{q_{3}}{q_{2}}=a_{3}+\frac{e_{2}}{a_{2}}, \frac{p_{1}}{p_{0}}=a_{1}$, $\frac{p_{2}}{p_{1}}=a_{2}+\frac{e_{1}}{a_{1}}, \frac{p_{3}}{p_{2}}=a_{3}+\frac{e_{2}}{a_{2}+\frac{e_{1}}{a_{1}}}$ and

$$
\begin{gathered}
\frac{q_{n}}{q_{n-1}}=a_{n}+\frac{e_{n-1}}{\frac{q_{n-1}}{q_{n-2}}}=a_{n}+\frac{e_{n-1}}{a_{n-1}+\frac{e_{n-2}}{\ddots \cdot+\frac{e_{2}}{a_{2}}}}, \quad \forall n \geq 2, \\
\frac{p_{n}}{p_{n-1}}=a_{n}+\frac{e_{n-1}}{\frac{p_{n-1}}{p_{n-2}}}=a_{n}+\frac{e_{n-1}}{a_{n-1}+\frac{e_{n-2}}{\ddots \cdot+\frac{e_{1}}{a_{1}}}}, \quad \forall n \geq 1 .
\end{gathered}
$$

The latter and Lemma 10 yield

$$
-\frac{1}{\left[\overline{\left(a_{1}, e_{1}\right), \ldots,\left(a_{n}, e_{n}\right)}\right]^{*}}=\frac{e_{n} p_{n}}{p_{n-1}}, \quad \forall n \geq 1 .
$$

Given $N \in \mathbb{N}$ and $\alpha, \beta, \beta_{1}, \beta_{2} \geq 1$, we introduce the sets

$$
\begin{aligned}
W_{E} & :=\{(a, e) \mid a \in 2 \mathbb{N}, e \in\{ \pm 1\}\}, \\
\mathscr{W}_{E} & :=\left\{\left(w_{1}, \ldots, w_{m}\right) \mid m \geq 1, w_{i} \in W_{E}\right\}, \\
\mathscr{W}_{E}^{+} & :=\left\{\left(w_{1}, \ldots, w_{m}\right) \in \mathscr{W}_{E} \mid\left(-e_{1}\right) \cdots\left(-e_{m}\right)=+1\right\}, \\
\mathscr{S}_{+} & :=\{\sigma \in \mathscr{S} \mid \operatorname{det}(\sigma)=+1\}, \\
\mathscr{S}(\alpha, \beta ; N) & :=\left\{\sigma \in \mathscr{S}_{+} \mid \operatorname{Tr}(\sigma) \leq N, p \geq \alpha q, p^{\prime} \geq \beta p\right\}, \quad \mathscr{S}(N):=\mathscr{S}(1,1 ; N), \\
\mathscr{T}\left(\alpha, \beta_{1}, \beta_{2} ; N\right) & :=\left\{(\omega, k) \mid \omega \in \mathscr{R}_{E}, \omega \geq \alpha, \omega^{*} \in\left[-\frac{1}{\beta_{2}}, \frac{1}{\beta_{1}}\right], \operatorname{Tr}\left(\widetilde{\Omega}(\omega)^{k}\right) \leq N, k \in \mathbb{N}\right\}, \\
\mathscr{T}_{k}\left(\alpha, \beta_{1}, \beta_{2} ; N\right) & :=\left\{(\omega, k) \mid \omega \in \mathscr{R}_{E}, \omega \geq \alpha, \omega^{*} \in\left[-\frac{1}{\beta_{2}}, \frac{1}{\beta_{1}}\right], \operatorname{Tr}\left(\widetilde{\Omega}(\omega)^{k}\right) \leq N\right\}, \quad k \in \mathbb{N}, \\
\mathscr{T}(N) & :=\mathscr{T}(1,1,1 ; N), \quad \mathscr{T}_{k}(N):=\mathscr{T}_{k}(1,1,1 ; N) .
\end{aligned}
$$

For given $N, \alpha, \beta_{1}, \beta_{2}$, the sets $\mathscr{T}_{k}\left(\alpha, \beta_{1}, \beta_{2} ; N\right)$ are disjoint. Therefore

$$
\left|\mathscr{T}\left(\alpha, \beta_{1}, \beta_{2} ; N\right)\right|=\sum_{k \geq 1}\left|\mathscr{T}_{k}\left(\alpha, \beta_{1}, \beta_{2} ; N\right)\right|, \quad \forall N \geq 1 .
$$

We have already proved that the map

$$
\beta_{E}: \mathscr{W}_{E} \rightarrow \mathscr{S}, \quad \beta_{E}\left(\left(a_{1}, e_{1}\right), \ldots,\left(a_{m}, e_{m}\right)\right):=M\left(a_{1}, e_{1}\right) \cdots M\left(a_{m}, e_{m}\right)
$$

is well defined and onto. Define also the sets

$$
\begin{aligned}
\mathscr{W}_{E}^{+}(N) & :=\left\{w \in \mathscr{W}_{E}^{+} \mid \operatorname{Tr}\left(\beta_{E}(w)\right) \leq N\right\}, \\
\mathscr{W}_{E}^{+}\left(\alpha, \beta_{1}, \beta_{2} ; N\right) & :=\left\{w=\left(w_{1}, \ldots, w_{m}\right) \in \mathscr{W}_{E}^{+}(N) \mid \omega:=\left[\overline{w_{1}, \ldots, w_{m}}\right] \geq \alpha, \omega^{*} \in\left[-\frac{1}{\beta_{2}}, \frac{1}{\beta_{1}}\right]\right\} .
\end{aligned}
$$

Proposition 19. (i) The map $\beta_{E}$ is one-to-one and $\beta_{E}\left(\mathscr{W}_{E}^{+}(N)\right)=\mathscr{S}(N)$.

(ii) The map

$j_{E}: \mathscr{W}_{E}^{+}(N) \rightarrow \mathscr{T}(N), \quad j_{E}\left(\left(a_{1}, e_{1}\right), \ldots,\left(a_{m}, e_{m}\right)\right):=\left(\omega=\left[\overline{\left(a_{1}, e_{1}\right), \ldots,\left(a_{m}, e_{m}\right)}\right], \frac{m}{\operatorname{eper}(\omega)}\right)$ is a one-to-one correspondence.

Proof. To check that $\beta_{E}$ is one-to-one we employ a descending argument. Assuming

$$
M_{\left(a_{1}, e_{1}\right)} \cdots M_{\left(a_{n}, e_{n}\right)}=\left(\begin{array}{cc}
p_{n} & p_{n-1} e_{n} \\
q_{n} & q_{n-1} e_{n}
\end{array}\right)=M_{\left(b_{1}, f_{1}\right)} \cdots M_{\left(b_{k}, f_{k}\right)}=\left(\begin{array}{cc}
P_{k} & P_{k-1} f_{k} \\
Q_{k} & Q_{k-1} f_{k}
\end{array}\right),
$$


the previous remark implies $f_{k}=e_{n}$ and $\frac{Q_{k}}{Q_{k-1}}=\frac{q_{n}}{q_{n-1}}$. This gives in turn $b_{k}=a_{n}$, leading to $\left.M_{\left(a_{1}, e_{1}\right)} \cdots M_{\left(a_{n-1}, e_{n-1}\right)}=M_{\left(b_{1}, f_{1}\right)} \cdots M_{\left(b_{k-1}, f_{k-1}\right.}\right)$.

The equality $\beta_{E}\left(\mathscr{W}_{E}^{+}(N)\right)=\mathscr{S}(N)$ is clear.

(ii) First we check that $j_{E}\left(\mathscr{W}_{E}^{+}(N)\right) \subseteq \mathscr{T}(N)$. Let $w:=\left(\left(a_{1}, e_{1}\right), \ldots,\left(a_{m}, e_{m}\right)\right) \in \mathscr{W}_{E}^{+}(N)$ and $\omega:=\left[\overline{\left(a_{1}, e_{1}\right), \ldots,\left(a_{m}, e_{m}\right)}\right] \in \mathscr{R}_{E}$. Employing the notation introduced after Lemma 8 , let $n:=\operatorname{per}(\omega)$. Set $k:=\frac{m}{n} \in \mathbb{N}$. If $\delta_{n}=+1$, then $\ell:=\operatorname{eper}(\omega)=n$ and $\frac{m}{\ell}=k \in \mathbb{N}$. If $\delta_{n}=-1$, then $\ell=2 n$ and we have $1=\left(-e_{1}\right) \cdots\left(-e_{m}\right)=\delta_{n}^{k}=(-1)^{k}$, so $k$ is even and $\frac{m}{\ell}=\frac{m}{2 n}=\frac{k}{2} \in \mathbb{N}$. On the other hand $\beta_{E}(w)=\widetilde{\Omega}(\omega)^{m / \ell}$, showing $\operatorname{Tr}\left(\widetilde{\Omega}(\omega)^{m / \ell}\right) \leq N$, and therefore $j_{E}(w) \in \mathscr{T}(N)$.

The map $j_{E}$ is clearly one-to-one. If $w=\left(\left(a_{1}, e_{1}\right), \ldots,\left(a_{m}, e_{m}\right)\right), w^{\prime}=\left(\left(b_{1}, f_{1}\right), \ldots,\left(b_{s}, f_{s}\right)\right) \in$ $\mathscr{W}_{E}^{+}(N)$ and $j_{E}(w)=\left(\omega, \frac{m}{\operatorname{eper}(\omega)}\right)=j_{E}\left(w^{\prime}\right)=\left(\omega^{\prime}, \frac{s}{\operatorname{eper}\left(\omega^{\prime}\right)}\right)$, then $\omega=\omega^{\prime}$ and $m=s$, so clearly $a_{i}=b_{i}, i=1, \ldots, m$.

To check surjectivity of $j_{E}$, let $(\omega, k) \in \mathscr{T}(N), \omega=\left[\overline{w_{1}, \ldots, w_{n}}\right]$ with $w_{i}=\left(a_{i}, e_{i}\right)$ and $n=\operatorname{per}(\omega), \ell=\operatorname{eper}(\omega)$. Take $m:=k \ell$ and $w=\left(w_{1}, \ldots, w_{\ell}, \ldots, w_{1}, \ldots, w_{\ell}\right) \in \mathscr{W}_{E}^{+}$with the block $\left(w_{1}, \ldots, w_{\ell}\right)$ repeating $k$ times. Since $\beta_{E}(w)=\widetilde{\Omega}(\omega)^{k}$, it follows that $\operatorname{Tr}\left(\beta_{E}(w)\right) \leq N$. Therefore, $w \in \mathscr{W}_{E}^{+}(N)$ and $j_{E}(w)=\left(\omega, \frac{m}{\ell}\right)$.

Corollary 20. The restriction of $j_{E}$ gives a bijection between the sets $\mathscr{W}_{E}^{+}\left(\alpha, \beta_{1}, \beta_{2} ; N\right)$ and $\mathscr{T}\left(\alpha, \beta_{1}, \beta_{2} ; N\right)$.

\section{Even Continued fractions and the Pell EQUation}

In this section we discuss some connections between $E$-reduced QIs and the Pell equations $t^{2}-\Delta u^{2}= \pm 1$, extending some well known results about reduced QIs ([16, 25]). This part of the paper is not directly related to the proofs of Theorems 1 or 4 .

Throughout this section we consider $\omega \in \mathscr{R}_{E}(\Delta), \omega=\left[\overline{\left(a_{1}, e_{1}\right), \ldots,\left(a_{n}, e_{n}\right)}\right]>1$ with $n=$ $\operatorname{per}(\omega)$, and minimal polynomial $A X^{2}+B X+C$, where $A>0,(A, B, C)=1$, and $\Delta=$ $B^{2}-4 A C \equiv 0,1(\bmod 4)$. As $\left(\begin{array}{cc}p_{n} & p_{n-1} e_{n} \\ q_{n} & q_{n-1} e_{n}\end{array}\right) \omega=\omega$, one has

$$
\frac{q_{n}}{A}=\frac{q_{n-1} e_{n}-p_{n}}{B}=\frac{-p_{n-1} e_{n}}{C}=: v \in \mathbb{Z} .
$$

When $n$ is odd, both $q_{n}$ and $p_{n-1}$ are odd, while $q_{n-1} e_{n}-p_{n}$ is always even. It follows that $A, C, v$ are odd and $B$ must be even, so $\Delta \equiv 0(\bmod 4)$. Hence $\Delta \equiv 1(\bmod 4) \Longrightarrow \operatorname{per}(\omega)$ even.

Consider the abelian groups

$$
\begin{aligned}
& \mathscr{F}_{\Delta}:=\left\{t+u \sqrt{\Delta} \mid t, u \in \mathbb{Z}, t^{2}-\Delta u^{2}= \pm 1\right\} \quad \text { and } \\
& \mathscr{F}_{\Delta}^{+}:=\left\{t+u \sqrt{\Delta} \mid t>0, t^{2}-\Delta u^{2}=+1\right\} .
\end{aligned}
$$

It is well known that $\mathscr{F}_{-}^{+}$is an infinite cyclic subgroup of $\mathscr{F}_{\Delta}$.

Recall from Lemma 9 that the one-to-one group homomorphism $\Lambda_{\omega}^{E}: \widetilde{\Theta}_{\omega} \rightarrow \mathbb{R}$ maps a matrix $\left(\begin{array}{ll}a & b \\ c & d\end{array}\right)$ to its eigenvalue $c \omega+d$.

Lemma 21. (i) If $\Delta \equiv 1(\bmod 4)$, then $\Lambda_{\omega}^{E}\left(\widetilde{\Theta}_{\omega}\right)=\mathscr{F}_{\Delta}$. Furthermore, $\sigma \equiv I_{2}(\bmod 2)$ for every $\sigma \in \widetilde{\Theta}_{\omega}$.

(ii) If $\Delta=4 \Delta_{0}$ and $\Delta_{0}$ is not a perfect square, then $\Lambda_{\omega}^{E}\left(\widetilde{\Theta}_{\omega}\right) \subseteq \mathscr{F}_{\Delta_{0}}$. If in addition $\Delta_{0}$ is odd and $B \equiv 0(\bmod 4)$, then $\Lambda_{\omega}^{E}\left(\widetilde{\Theta}_{\omega}\right)=\mathscr{F}_{\Delta_{0}}$.

Proof. (i) The entries of a matrix $\sigma=\left(\begin{array}{ll}a & b \\ c & d\end{array}\right) \in \widetilde{\Theta}_{\omega}$ must satisfy $\frac{c}{A}=\frac{d-a}{B}=\frac{-b}{C}=v \in \mathbb{Z}$. We have $D:=(a-d)^{2}+4 b c=(a+d)^{2}-4 \operatorname{det}(\sigma)=v^{2} \Delta$. Since $a-d \equiv 0(\bmod 2)$ and $\Delta$ is odd, it follows 
that $D=4 u_{0}^{2} \Delta$ for some $u_{0} \in \mathbb{Z}$. Setting $t_{0}:=\frac{a+d}{2} \in \mathbb{Z}$, one has $c \omega+d=\frac{a+d+\sqrt{D}}{2}=t_{0}+u_{0} \sqrt{\Delta}$, with $\mathfrak{N}(c \omega+d)=t_{0}^{2}-\Delta u_{0}^{2}=\operatorname{det}(\sigma)= \pm 1$. This shows $\Lambda_{\omega}^{E}\left(\widetilde{\Theta}_{\omega}\right) \subseteq \mathscr{F}_{\Delta}$.

To show that $\Lambda_{\omega}^{E}$ is surjective, note first that $2 A \omega+B=\sqrt{\Delta}$. Let $t_{0}+u_{0} \sqrt{\Delta} \in \mathscr{F} \Delta$. Take $\sigma:=\left(\begin{array}{cc}t_{0}-B u_{0} & -2 C u_{0} \\ 2 A u_{0} & t_{0}+B u_{0}\end{array}\right) \in \mathrm{GL}(2, \mathbb{Z})$. We have $\left(t_{0}-B u_{0}\right)\left(t_{0}+B u_{0}\right)=t_{0}^{2}-\Delta u_{0}^{2}-4 A C y u_{0}^{2} \equiv 1$ $(\bmod 2)$, hence $\sigma \equiv I_{2}(\bmod 2)$, showing $\sigma \in \widetilde{\Theta}$. The equality $\sigma \omega=\omega$ is checked by direct verification. Finally, we see that $\Lambda_{\omega}^{E}(\sigma)=t_{0}+(2 A \omega+B) u_{0}=t_{0}+u_{0} \sqrt{\Delta}$.

(ii) In this case $D=u^{2} \Delta=4 u^{2} \Delta_{0}$ and $c \omega+d=\frac{a+d}{2}+u \sqrt{\Delta_{0}}=t_{0}+u_{0} \sqrt{\Delta}$ with $t_{0}^{2}-\Delta u_{0}^{2}=$ $\mathfrak{N}(c \omega+d)= \pm 1$, showing $c \omega+d \in \mathscr{F}_{\Delta_{0}}$.

Assume in addition $\Delta_{0}$ odd and $B \equiv 0(\bmod 4)$. Write $B=2 B_{0}, B_{0} \in \mathbb{Z}$. Let $t_{0}+u_{0} \sqrt{\Delta_{0}} \in$ $\mathscr{F}_{\Delta_{0}}$. Take $\sigma:=\left(\begin{array}{cc}t_{0}-B_{0} u_{0} & -C u_{0} \\ A u_{0} & t_{0}+B_{0} u_{0}\end{array}\right)$ with $\operatorname{det}(\sigma)=t_{0}^{2}-\frac{\Delta}{4} u_{0}^{2}=t_{0}^{2}-\Delta_{0} u_{0}^{2}= \pm 1$. Again, the equalities $\sigma \omega=\omega$ and $\Lambda_{\omega}^{E}(\sigma)=t_{0}+u_{0} \sqrt{\Delta_{0}}$ follow by direct verification, so it only remains to check that $\sigma \equiv I_{2}$ or $J_{2}(\bmod 2)$. For this, observe that since $\Delta_{0}$ is odd and $t_{0}^{2}-\Delta_{0} u_{0}^{2}= \pm 1$ one has $t_{0} \equiv u_{0}+1(\bmod 2)$. This shows that when $u_{0}$ is even, $t_{0}$ must be odd and so $\sigma \equiv I_{2}$ $(\bmod 2)$. When $u_{0}$ is odd, $t_{0}$ must be even, so $\sigma \equiv\left(\begin{array}{cc}-B_{0} & -C \\ A & B_{0}\end{array}\right)(\bmod 2)$. But $B_{0}^{2}-A C=\Delta_{0}$ is odd and $B_{0}$ is even, hence $A C$ is odd and consequently $\sigma \equiv J_{2}(\bmod 2)$.

The following statement ([6, Proposition 3.2], see also [22]) will be useful. The formulation given here on $[1, \infty)$ is immediately transported from the one on $[0,1]$ by conjugating by the matrix $\left(\begin{array}{ll}0 & 1 \\ 1 & 0\end{array}\right)$.

Lemma 22. Let $u \in[1, \infty) \backslash \mathbb{Q}$ and $\sigma=\left(\begin{array}{ll}a & b \\ c & d\end{array}\right) \in \widetilde{\Theta}$. The following are equivalent:

(i) There exists $m \geq 1$ such that $\sigma=\left(\begin{array}{c}p_{m}(u) p_{m-1}(u) e \\ q_{m}(u) q_{m-1}(u) e\end{array}\right)$ for some $e \in\{ \pm 1\}$.

(ii) $\sigma \in \mathscr{R}_{E}^{+}(u) \cup \mathscr{R}_{E}^{-}(u)$, where

$$
\mathscr{R}_{E}^{\varepsilon}(u):=\left\{\sigma \in \widetilde{\Theta} \mid \begin{array}{c}
a \geq c>\varepsilon d \geq 0 \\
\varepsilon b \geq \varepsilon d \geq 0
\end{array} \text { and }\left|\frac{d u-b}{-c u+a}\right|>1\right\}, \quad \varepsilon \in\{ \pm 1\} .
$$

We also have $e=\operatorname{sgn}(b)=\operatorname{sgn}(d)$.

Lemma 23. Suppose $\omega=\left[\overline{\left(a_{1}, e_{1}\right), \ldots,\left(a_{n}, e_{n}\right)}\right]>1$, where $n=\operatorname{per}(\omega)$. Then

(i) If $\sigma=\left(\begin{array}{ll}a & b \\ c & d\end{array}\right) \in \widetilde{\Theta}_{\omega}$ and $c \geq d \geq 0$, then $e_{n}=+1$ and $\sigma=\Omega_{E}(\omega)^{k}$ for some $k \in \mathbb{N}$.

(ii) If $\sigma=\left(\begin{array}{l}a-b \\ c-d\end{array}\right) \in \widetilde{\Theta}_{\omega}$ and $c \geq d \geq 0$, then $e_{n}=-1$ and $\sigma=\Omega_{E}(\omega)^{k}$ for some $k \in \mathbb{N}$.

Proof. In both cases we have $\sigma^{-1} \omega=\left(\begin{array}{cc} \pm d & \mp b \\ -c & a\end{array}\right) \omega= \pm \frac{d \omega-b}{-c \omega+a}=\omega>1$, showing $\left|\frac{d \omega-b}{-c \omega+a}\right|=\omega>1$. Note also that $c-d \equiv 1(\bmod 2)$, so $c>d \geq 1$.

(i) From $\left|\omega-\frac{a}{c}\right|=\left|\frac{a \omega+b}{c \omega+d}-\frac{a}{c}\right|=\frac{1}{c(c \omega+d)}<\frac{1}{c}$ we infer $|c \omega-a|<1$. This shows $c-a<c \omega-a<1$, so $a \geq c \geq 2$. On the other hand, $b \geq \frac{a d-1}{c} \geq d-\frac{1}{c}>d-1$ gives $b \geq d$. By Lemma 22 ,

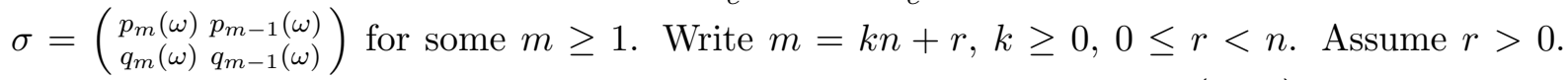
Rewriting the previous equality as $\sigma=\Omega_{E}(\omega)^{k} M\left(a_{1}, e_{1}\right) \cdots M\left(a_{r}, e_{r}\right)\left(\begin{array}{cc}1 & 0 \\ 0 & e_{m}\end{array}\right)$ and employing $\Omega_{E}(\omega)^{-k} \sigma \omega=\omega$, we get $M\left(a_{r}, e_{r}\right)^{-1} \cdots M\left(a_{1}, e_{1}\right)^{-1} \omega=e_{m} \omega$, so $\left[\left(a_{r+1}, e_{r+1}\right), \ldots,\left(a_{n}, e_{n}\right), \omega\right]=$ $e_{m} \omega>1$. This gives $e_{m}=+1$ and $\omega=\left[\left(a_{1}, e_{1}\right), \ldots,\left(a_{r}, e_{r}\right), \omega\right]$, contradiction. It follows that $r=0$, so $\sigma=\Omega_{E}(\omega)^{k}$. This also gives $e_{n}=e_{k n}=e_{m}=+1$.

(ii) Similar to (i), employ $c \omega-d>c-d \geq 1$ and $\omega=\frac{a \omega-b}{c \omega-d}$ to get $a \geq c \geq 2$ and $b \geq d$. By Lemma 22, $\sigma=\left(\begin{array}{c}p_{m}(\omega)-p_{m-1}(\omega) \\ q_{m}(\omega)-q_{m-1}(\omega)\end{array}\right)$ for some $m=k n+r \geq 1,0 \leq r<n$. As in (i), when 
$r>0$ this gives $\sigma=\Omega_{E}(\omega)^{k} M\left(a_{1}, e_{1}\right) \cdots M\left(a_{r}, e_{r}\right)\left(\begin{array}{cc}1 & 0 \\ 0 & -e_{m}\end{array}\right)$, leading in turn to $e_{m}=-1$ and $\omega=\left[\left(a_{1}, e_{1}\right), \ldots,\left(a_{r}, e_{r}\right), \omega\right]$, contradiction. Hence $r=0, \sigma=\Omega_{E}(\omega)^{k}$ and $e_{n}=-1$.

With $\omega$ and $n$ as in Lemma 23, the number

$$
\epsilon:=\Lambda_{\omega}^{E}\left(\Omega_{E}(\omega)\right)=q_{n} \omega+q_{n-1} e_{n}
$$

can be expressed as

$$
\epsilon=\frac{p_{n}+q_{n-1} e_{n}+\sqrt{\left(p_{n}+q_{n-1} e_{n}\right)^{2}-4 \delta_{n}}}{2} \in \mathscr{F}_{\Delta},
$$

where $\delta_{n}:=\left(-e_{1}\right) \cdots\left(-e_{n}\right)$. Since $p_{n}+q_{n-1} e_{n}>0, \forall n \geq 1$, we have

$$
\mathfrak{N}(\epsilon)=1 \Longleftrightarrow\left(-e_{1}\right) \cdots\left(-e_{n}\right)=1 \Longleftrightarrow \epsilon \in \mathscr{F}_{\Delta}^{+} .
$$

Lemma 24. Suppose that $\Delta \equiv 1(\bmod 4)$ and let $\sigma \in \widetilde{\Theta}_{\omega} 1$ with $\Lambda_{\omega}^{E}(\sigma)>1$. Then $\Lambda_{\omega}^{E}(\sigma)=\epsilon^{k}$ for some $k \geq 1$. In particular, if $n$ is even and $\operatorname{eper}(\omega)=\operatorname{per}(\omega)$, then $\epsilon=\Lambda_{\omega}^{E}\left(\Omega_{E}(\omega)\right)$ is the fundamental unit of $\mathscr{F}_{\Delta}^{+}$.

Proof. Let $\sigma=\left(\begin{array}{ll}a & b \\ c & d\end{array}\right) \in \widetilde{\Theta}_{\omega}$ with $\eta:=c \omega+d>1$. Since $\mathfrak{N}(\eta)=\eta \eta^{*}=\operatorname{det}(\sigma)= \pm 1$, we have $\left|\eta^{*}\right|=\left|c \omega^{*}+d\right|<1$, leading to $\eta-\eta^{*}=c\left(\omega-\omega^{*}\right)>0$, and so $c \geq 1$. On the other hand $\omega^{*} \in(-1,1)$ yields $-c+d<\eta^{*}=c \omega^{*}+d<c+d$, showing $-c \leq d \leq c$. Actually we have $-c<d<c$ because $c-d \equiv 1(\bmod 2)$. Two situations can occur:

(a) $d=0$, leading to $c=1$ (so $\eta=\omega)$ and $b= \pm 1$. We get $a+\frac{ \pm 1}{\omega}=\omega$, giving $a>1$ and $\omega=[\overline{(a, \pm 1)}]$ with $\epsilon=q_{1} \omega+q_{0}=\omega=\eta$.

(b) $c> \pm d \geq 1$, leading upon Lemma 23 to $e_{n}= \pm 1$ and $\sigma=\Omega_{E}(\omega)^{k}, k \geq 1$. Therefore we get $\eta=\Lambda_{\omega}^{E}(\sigma)=\Lambda_{\omega}^{E}\left(\Omega_{E}(\omega)^{k}\right)=\Lambda_{\omega}^{E}\left(\Omega_{E}(\omega)\right)^{k}=\epsilon^{k}$.

Remark 25. Lemma 24 also works when $\Delta \equiv 0(\bmod 4)$ : if $\left(-e_{1}\right) \cdots\left(-e_{n}\right)=1$, then $\epsilon$ is the generator of the (infinite cyclic) group $\Lambda_{\omega}^{E}\left(\widetilde{\Theta}_{\omega}\right)^{+}:=\left\{\Lambda_{\omega}^{E}(\sigma) \mid \sigma \in \widetilde{\Theta}_{\omega}, \operatorname{det}(\sigma)=1, \operatorname{Tr}(\sigma)>0\right\}$. When $\Delta=4 \Delta_{0}, \Delta_{0}$ odd and $B \equiv 0(\bmod 4)$, we also have $\Lambda_{\omega}^{E}\left(\widetilde{\Theta}_{\omega}\right)^{+}=\mathscr{F}_{\Delta_{0}}^{+}$.

\section{BACKWARD CONTINUED FRACTIONS}

This section is concerned with backward continued fractions and $B$-reduced QIs. We consider $\llbracket a_{1}, a_{2}, a_{3}, \ldots \rrbracket$ as in equation $(1.10)$. The Gauss shift $T_{B}$ acts on $[1, \infty) \backslash \mathbb{Q}$ as $T_{B}\left(\llbracket a_{1}, a_{2}, a_{3}, \ldots \rrbracket\right)=$ $\llbracket a_{2}, a_{3}, \ldots \rrbracket$, or, in different notation,

$$
T_{B}(u)=M\left(a_{1},-1\right)^{-1} u=\left(\begin{array}{cc}
0 & -1 \\
1 & -a_{1}
\end{array}\right) u=\frac{1}{a_{1}-u}=\frac{1}{1-\{u\}},
$$

where $a_{1}=a_{1}(u)=1+\lfloor u\rfloor \geq 2$. The natural extension of the endomorphism $T_{B}$ is the automorphism of $[1, \infty) \times[0,1]$ given by

$$
\widetilde{T}_{B}(u, v)=\left(T_{B}(u), \frac{1}{a_{1}-v}\right)=\left(\frac{1}{a_{1}-u}, \frac{1}{a_{1}-v}\right),
$$

acting on the digits as a two-sided shift:

$$
\widetilde{T}_{B}\left(\llbracket a_{1}, a_{2} \ldots \rrbracket, \llbracket a_{0}, a_{-1}, \ldots \rrbracket^{-1}\right)=\left(\llbracket a_{2}, a_{3}, \ldots \rrbracket, \llbracket a_{1}, a_{0}, a_{-1}, \ldots \rrbracket^{-1}\right), \quad a_{i} \in \mathbb{N}, a_{i} \geq 2 .
$$

\footnotetext{
${ }^{1}$ Recall that in this case $n$ must be even and $\sigma \equiv I_{2}(\bmod 2)$.
} 

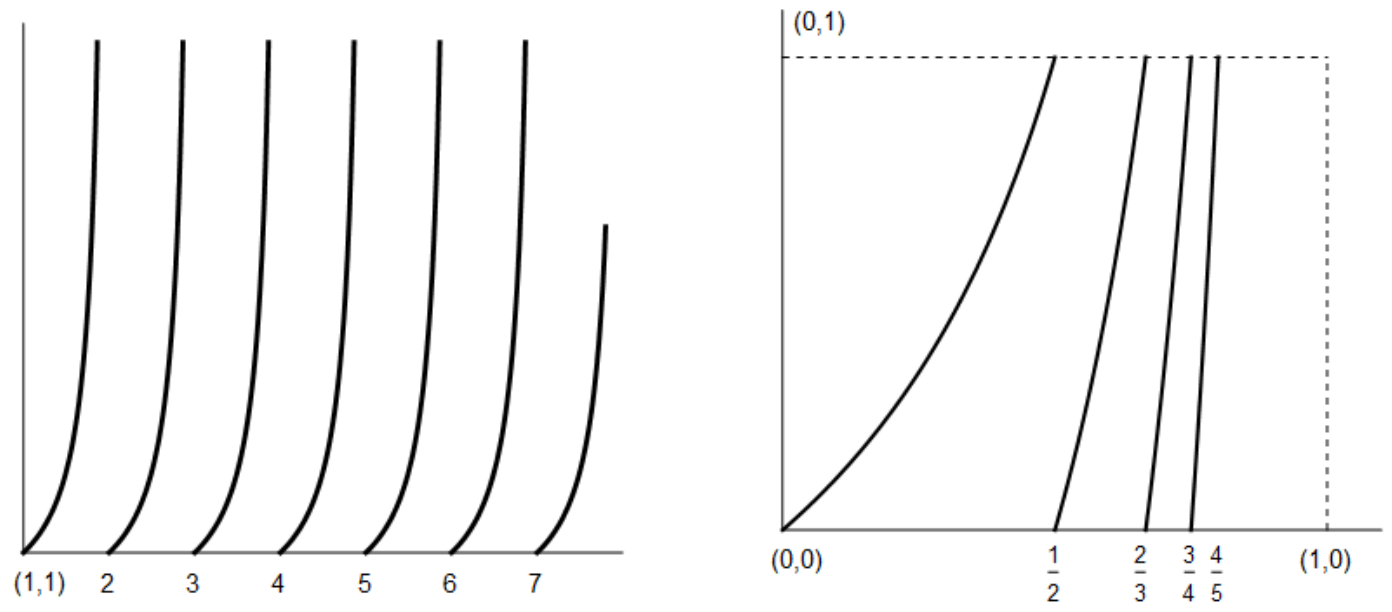

Figure 2. The graphs of the maps $T_{B}$ and $\bar{T}_{B}$

From general ergodic theoretical considerations ([1]), the infinite measure $(u-v)^{-2} d u d v$ is $\widetilde{T}_{B}$-invariant, while $d \mu_{B}=\left(\int_{0}^{1}(u-v)^{-2} d v\right) d u=\frac{d u}{u(u-1)}$ is $T_{B}$-invariant.

Conjugating through $J_{B}(x):=\frac{1}{1-x}$, one gets the Rényi-Gauss map $\bar{T}_{B}:=J_{B}^{-1} T_{B} J_{B}$, which acts on $[0,1]$ as in equation (1.11) with invariant measure $\nu_{B}=J_{B *} \mu_{B}=\frac{d x}{x}([29$, 1]). The endomorphism $\bar{T}_{B}$ is exact in Rohlin's sense, and $\nu_{B}$ is the unique $\sigma$-finite Lebesgue absolutely continuous $\bar{T}_{B}$-invariant measure.

Given $a_{i} \geq 2$, define $\frac{p_{0}}{q_{0}}:=\frac{1}{0}, \frac{p_{1}}{q_{1}}:=\frac{a_{1}}{1}, \frac{p_{2}}{q_{2}}:=a_{1}-\frac{1}{a_{2}}=\frac{a_{1} a_{2}-1}{a_{2}}$, and

$$
\frac{p_{k}}{q_{k}}:=\frac{a_{k} p_{k-1}-p_{k-2}}{a_{k} q_{k-1}-q_{k-2}}=a_{1}-\frac{1}{a_{2}-\frac{1}{\ddots \cdot-\frac{1}{a_{k}}}}, \quad \forall k \geq 2 .
$$

All algebraic computations with even continued fractions apply to backward continued fractions taking $e_{i}=-1, \forall i \geq 1$. In particular, as in Remark 18 we have

$$
\begin{gathered}
\frac{q_{n}}{q_{n-1}}=a_{n}-\frac{1}{\frac{q_{n-1}}{q_{n-2}}}=a_{n}-\frac{1}{a_{n-1}-\frac{1}{\ddots \cdot-\frac{1}{a_{2}}}}, \quad \forall n \geq 2, \\
\frac{p_{n}}{p_{n-1}}=a_{n}-\frac{1}{\frac{p_{n-1}}{p_{n-2}}}=a_{n}-\frac{1}{a_{n-1}-\frac{1}{\ddots \cdot-\frac{1}{a_{1}}}}, \quad \forall n \geq 1 .
\end{gathered}
$$

We also have

$$
p_{k}>q_{k}>q_{k-1}, \quad p_{k}>p_{k-1}>q_{k-1}
$$

and quite importantly,

$$
p_{k} q_{k-1}-p_{k-1} q_{k}=-1, \quad \forall k \geq 1 .
$$

This shows that the sequence $\left(\frac{p_{k}}{q_{k}}\right)$ is decreasing and $u:=\lim _{k} \frac{p_{k}}{q_{k}} \in[1, \infty) \backslash \mathbb{Q}$ satisfies

$$
p_{k}-q_{k} u>0, \quad \forall k \geq 0 \text {. }
$$


In the opposite direction, the digits of $u$ are captured by $a_{1}=a_{1}(u):=1+\lfloor u\rfloor$ and

$$
a_{n+1}=a_{n+1}(u)=a_{1}\left(T_{B}^{n}(u)\right)=1+\left\lfloor T_{B}^{n}(u)\right\rfloor .
$$

The corresponding ECF formulas with $e_{i}=-1, \forall i$ provide

$$
T_{B}^{k}(u)=\frac{p_{k-1}-q_{k-1} u}{p_{k}-q_{k} u}, \quad \forall k \geq 1 .
$$

This gives

$$
u=\frac{T_{B}^{k}(u) p_{k}-p_{k-1}}{T_{B}^{k}(u) q_{k}-q_{k-1}}, \quad \forall k \geq 1
$$

and so

$$
T_{B}(u) T_{B}^{2}(u) \cdots T_{B}^{k}(u)=\frac{1}{p_{k}-q_{k} u}, \quad \forall k \geq 1 .
$$

Definition 26. The quadratic irrational $\omega>1$ is called $B$-reduced if $0<\omega^{*}<1$. We denote

$$
\mathscr{R}_{B}(\Delta):=\{\omega \in \mathscr{X}(\Delta) \mid \omega B \text {-reduced }\} \quad \text { and } \quad \mathscr{R}_{B}:=\bigcup_{\Delta>0} \mathscr{R}_{B}(\Delta) .
$$

Exactly as in Proposition 14 one shows

Proposition 27. For every quadratic irrational $\omega>1$, the following are equivalent:

(i) $\omega \in \mathscr{R}_{B}$.

(ii) $B C F(\omega)$ is periodic, i.e. $\omega=\llbracket \overline{a_{1}, \ldots, a_{n}} \rrbracket$.

(iii) $\omega$ is a periodic point of the map $T_{B}$.

For every $u \in[1, \infty) \backslash \mathbb{Q}$ consider the set

$$
\mathscr{M}_{B}(u):=\left\{\sigma=\left(\begin{array}{ll}
p^{\prime} & -p \\
q^{\prime} & -q
\end{array}\right) \in \mathrm{SL}(2, \mathbb{Z}) \mid \begin{array}{c}
p^{\prime}>q^{\prime}>q>0, p^{\prime}>p>q \\
p^{\prime}-q^{\prime} u>0, E_{\sigma}(u):=\frac{p-q u}{p^{\prime}-q^{\prime} u}>1
\end{array}\right\} .
$$

Although the following statement is similar with Lemma 22, we provide a proof for the convenience of the reader.

Lemma 28. Let $u \in[1, \infty) \backslash \mathbb{Q}$. For every $\sigma=\left(\begin{array}{c}p^{\prime}-p \\ q^{\prime}-q\end{array}\right) \in \mathrm{SL}(2, \mathbb{Z})$, the following are equivalent:

(i) $\sigma \in \mathscr{M}_{B}(u)$.

(ii) $\frac{p^{\prime}}{q^{\prime}}$ and $\frac{p}{q}$ are consecutive $B C F(u)$-convergents.

Proof. (ii) $\Longrightarrow$ (i) Suppose $\frac{p}{q}=\frac{p_{n}}{q_{n}}=\frac{p_{n}(u)}{q_{n}(u)}$. Then 4.1)-4.4 imply $\sigma \in \mathscr{M}_{B}(u)$.

(i) $\Longrightarrow$ (ii) We prove by induction that the proposition

$$
\begin{aligned}
P(m):="(\forall u \in[1, \infty) \backslash \mathbb{Q}) & \left(\forall \sigma_{0}=\left(\begin{array}{ll}
p & -p_{0} \\
q & -q_{0}
\end{array}\right) \in \mathscr{M}_{B}(u), p_{0}<m\right) \\
& \left(\frac{p}{q} \text { and } \frac{p_{0}}{q_{0}} \text { are consecutive } B C F(u) \text {-convergents }\right) "
\end{aligned}
$$

holds for every $m \geq 2$.

When $m=2$ we get $p_{0}=1, q_{0}=0, q=1$, so $\sigma_{0}=\left(\begin{array}{cc}p & -1 \\ 1 & 0\end{array}\right)$. Since $E_{\sigma_{0}}(u)=\frac{1}{p-u}>1$ and $p-u=p-q u>0$, we infer $p>u>p-1$. This shows that $u=p-\frac{1}{\ddots}$, hence $\frac{p}{q}=\frac{p}{1}=\frac{p_{1}}{q_{1}}$ and $\frac{p_{0}}{q_{0}}=\frac{1}{0}$ are consecutive $B C F(u)$-convergents.

Suppose $P(m)$ holds for every $2 \leq m \leq m_{0}$. Let $\sigma=\left(\begin{array}{c}p^{\prime}-p \\ q^{\prime}-q\end{array}\right) \in \mathscr{M}_{B}(u)$ with $p=m_{0}$. Then $a:=1+\left\lfloor\frac{p^{\prime}}{p}\right\rfloor \geq 2$. Let $p_{0}:=a p-p^{\prime}, q_{0}:=a q-q^{\prime}$. From $a-1 \leq \frac{p^{\prime}}{p}<a$ we get $p \geq p_{0}=a p-p^{\prime}>0$. 
Actually $p>p_{0}$ because $p=p_{0}$ would imply $p \mid p^{\prime}$, thus contradicting $\left(p, p^{\prime}\right)=1$. So $p>p_{0}>0$. Since $\left(p, p^{\prime}\right)=1$ we also have $a-1<\frac{p^{\prime}}{p}$, hence $q_{0}=a q-q^{\prime}<\left(\frac{p^{\prime}}{p}+1\right) q-q^{\prime}=\frac{p^{\prime} q+p q-p q^{\prime}}{p}=$ $\frac{p q-1}{p}<q$. On the other hand $q_{0}>\frac{p^{\prime}}{p} q-q^{\prime}=-\frac{1}{p} \geq-\frac{1}{2}$, showing $q>q_{0} \geq 0$. The inequality $p_{0}>q_{0}$ follows from $p_{0}-q_{0}=a(p-q)-\left(p^{\prime}-q^{\prime}\right)>\frac{p^{\prime}}{p}(p-q)-\left(p^{\prime}-q^{\prime}\right)=\frac{p q^{\prime}-p^{\prime} q}{p}=\frac{1}{p}>0$, while $p^{\prime}-q^{\prime} u>0$ and $\frac{p^{\prime}}{q^{\prime}}<\frac{p}{q}$ yield $p-q u>0$.

Finally, $E_{\sigma_{0}}(u)=\frac{p_{0}-q_{0} u}{p-q u}>1$ is equivalent to $u>\frac{p-p_{0}}{q-q_{0}}$. The latter holds because $E_{\sigma}(u)=$ $\frac{p-q u}{p^{\prime}-q^{\prime} u}>0$ and $p^{\prime}-q^{\prime} u>0$ entail $u>\frac{p^{\prime}-p}{q^{\prime}-q}$, while $\frac{p^{\prime}-p}{q^{\prime}-q} \geq \frac{p-p_{0}}{q-q_{0}}=\frac{p^{\prime}-(a-1) p}{q^{\prime}-(a-1) q}$ is equivalent to the manifestly true $(a-2)\left(p^{\prime} q-p q^{\prime}\right)=2-a \leq 0$.

As a result of $(4.2)$, here we always have $\widetilde{\Omega}_{B}(\omega)=\Omega_{B}(\omega)$. The $B$-reduced quadratic irrationals $\omega$ will hereby be ordered by the spectral radius of the matrix $\Omega_{B}(\omega)$. A precise asymptotic formula for the cardinality of the set

$$
\mathscr{S}_{B}(\alpha, \beta ; N):=\left\{\left(\begin{array}{ll}
p^{\prime} & -p \\
q^{\prime} & -q
\end{array}\right) \in \mathrm{SL}(2, \mathbb{Z}) \mid \begin{array}{c}
p \geq \alpha q, p^{\prime} \geq \beta p \\
p^{\prime}>q^{\prime}>q \geq 0, p^{\prime}-q \leq N
\end{array}\right\}, \quad \alpha, \beta \geq 1,
$$

as $N \rightarrow \infty$, will be proved in Section 6. Finally, the approximation arguments detailed in Section 8 in the ECF situation will apply ad litteram to the BCF situation, where all $e_{i}$ 's are equal to -1 . This will allow us to conclude that $\left|\mathscr{S}_{B}(\alpha, \beta ; N)\right|$ provides an accurate approximation of $r_{B}(\alpha, \beta ; R)$, where $e^{R}=N^{2} \rightarrow \infty$, concluding the proof of Theorem 4 .

\section{Some estimates involving Euler's totient sums}

To derive asymptotic estimates for the number of $B$-reduced and $E$-reduced quadratic irrationals $\omega$ with $\left(\omega, \omega^{*}\right) \in[\alpha, \infty) \times\left[-\frac{1}{\beta_{2}}, \frac{1}{\beta_{1}}\right]$ we will need some detailed number theoretical estimates involving Euler's totient function. We consider the following sums:

$$
\begin{aligned}
& S_{0}(N):=\sum_{m \leq N} \varphi(m)=\frac{N^{2}}{2 \zeta(2)}+O(N \log N), \\
& S_{0}^{O}(N):=\sum_{\substack{m \leq N \\
m \text { odd }}} \varphi(m)=\sum_{a \leq \frac{N}{2}} \varphi(4 a), \quad S_{0}^{E}(N):=\sum_{\substack{m \leq N \\
m \text { even }}} \varphi(2 m), \\
& S_{1}(N):=\sum_{m \leq N} \frac{\varphi(m)}{m}=\frac{N}{\zeta(2)}+O(\log N), \\
& S_{1}^{O}(N):=\sum_{\substack{m \leq N \\
m \text { odd }}} \frac{\varphi(m)}{m}=\sum_{a \leq \frac{N}{2}} \frac{\varphi(4 a)}{2 a}, \quad S_{1}^{E}(N):=\sum_{\substack{m \leq N \\
m \text { even }}} \frac{\varphi(2 m)}{m}, \\
& S_{2}(N):=\sum_{m \leq N} \frac{\varphi(m)}{m^{2}}=\frac{1}{\zeta(2)}\left(\log N+\gamma-\frac{\zeta^{\prime}(2)}{\zeta(2)}\right)+O\left(N^{-1} \log N\right), \\
& S_{2}^{O}(N):=\sum_{\substack{m \leq N \\
m \text { odd }}} \frac{\varphi(m)}{m^{2}}, \quad S_{2}^{E}(N):=\sum_{\substack{m \leq N \\
m \text { even }}} \frac{\varphi(2 m)}{m^{2}}=\sum_{a \leq \frac{N}{2}} \frac{\varphi(4 a)}{4 a^{2}} .
\end{aligned}
$$

The estimates for $S_{0}(N)$ and $S_{1}(N)$ are well known. A proof of estimate for $S_{2}(N)$ can be found in [3, Cor. 4.5] (see also Chapter 3 in [35]). It relies essentially on some version of Perron's integral formula. Estimates for $S_{2}^{O}(N)$ and $S_{2}^{E}(N)$ are derived in analogous manner here, but require additional care. 
For every positive integer $\ell$, define

$$
C(\ell):=\frac{\varphi(\ell)}{\zeta(2) \ell} \prod_{p \mid \ell}\left(1-\frac{1}{p^{2}}\right)^{-1}
$$

with $C(2)=C(4)=\frac{2}{3 \zeta(2)}$. By Lemmas 2.1 and 2.2 in [4] we infer

$$
\begin{gathered}
S_{0}^{O}(N)=\frac{C(2) N^{2}}{2}+O(N \log N)=\frac{N^{2}}{3 \zeta(2)}+O(N \log N), \\
S_{0}^{E}(N)=\sum_{a \leq \frac{N}{2}} \varphi(4 a)=\frac{4 C(4) N^{2}}{8}+O(N \log N)=\frac{N^{2}}{3 \zeta(2)}+O(N \log N), \\
S_{1}^{O}(N)=C(2) N+O\left(\log ^{2} N\right)=\frac{2 N}{3 \zeta(2)}+O\left(\log ^{2} N\right), \\
S_{1}^{E}(N)=\sum_{a \leq \frac{N}{2}} \frac{\varphi(4 a)}{2 a}=\frac{4 C(4) N}{4}+O\left(\log ^{2} N\right)=\frac{2 N}{3 \zeta(2)}+O\left(\log ^{2} N\right) .
\end{gathered}
$$

To estimate $S_{2}^{O}(N)$ and $S_{2}^{E}(N)$, we follow closely the proof of [3, Lemma 4.4] and [35, Chapter 3]. We will first estimate the sums

$$
\widetilde{S}_{2}^{O}(N):=\sum_{\substack{m \leq N \\ m \text { odd }}} \frac{\varphi(m)}{m^{2}}(N-m)^{2} \quad \text { and } \quad \widetilde{S}_{2}^{E}(N):=\sum_{\substack{m \leq N \\ m \equiv 0}} \frac{\varphi(m)}{m^{2}}(N-m)^{2},
$$

employing the Perron formula

$$
\frac{1}{\pi i} \int_{\sigma_{0}-i \infty}^{\sigma_{0}+i \infty} \frac{y^{s}}{s(s+1)(s+2)} d s=\left\{\begin{array}{ll}
0 & \text { if } 0 \leq y \leq 1 \\
\left(1-y^{-1}\right)^{2} & \text { if } y \geq 1
\end{array} \quad\left(\sigma_{0}>0\right)\right.
$$

We consider the Dirichlet series

$$
\begin{aligned}
\zeta_{O}(s) & :=\sum_{\substack{m=1 \\
m \text { odd }}}^{\infty} \frac{1}{m^{s}}=\frac{1}{1+\sum_{k=1}^{\infty} \frac{\varphi\left(2^{k}\right)}{2^{k s}}} \prod_{p}\left(1+\sum_{k=1}^{\infty} \frac{\varphi\left(p^{k}\right)}{p^{k s}}\right) \\
& =\frac{2^{s}-2}{2^{s}-1} \cdot \frac{\zeta(s-1)}{\zeta(s)} \text { and } \\
\zeta_{E}(s) & :=\sum_{m=0}^{\infty} \frac{1}{m^{s}}=\frac{\sum_{k=2}^{\infty} \frac{\varphi\left(2^{k}\right)}{2^{k s}}}{1+\sum_{k=1}^{\infty} \frac{\varphi\left(2^{k}\right)}{2^{k s}}} \prod_{p}\left(1+\sum_{k=1}^{\infty} \frac{\varphi\left(p^{k}\right)}{p^{k s}}\right) \\
= & \frac{1}{2^{s-1}\left(2^{s}-1\right)} \cdot \frac{\zeta(s-1)}{\zeta(s)}(\operatorname{Re} s>2) .
\end{aligned}
$$

Employing the Perron formula with $y=\frac{N}{m}$ and (5.6), we infer

$$
\begin{aligned}
\widetilde{S}_{2}^{O}(N) & :=\sum_{\substack{m \leq N \\
m \text { odd }}} \varphi(m) \frac{N^{2}}{m^{2}}\left(1-\frac{m}{N}\right)^{2} \\
& =\frac{1}{\pi i} \int_{\sigma_{0}-i \infty}^{\sigma_{0}+i \infty} \sum_{\substack{m=1 \\
m \text { odd }}}^{\infty} \frac{\varphi(m)}{m^{s+2}} \cdot \frac{N^{s+2}}{s(s+1)(s+2)} d s=\frac{1}{2 \pi i} \int_{\sigma_{0}-i \infty}^{\sigma_{0}+i \infty} g_{N}^{O}(s) d s, \quad\left(\sigma_{0}>0\right)
\end{aligned}
$$


where

$$
\begin{aligned}
g_{N}^{O}(s) & =\frac{2 N^{s+2}}{s(s+1)(s+2)} \cdot \frac{2^{s+2}-2}{2^{s+2}-1} \cdot \frac{\zeta(s+1)}{\zeta(s+2)} d s \\
& =\frac{2 N^{s+2}\left(2^{s+2}-2\right)}{(s+1)(s+2)\left(2^{s+2}-1\right) \zeta(s+2)}\left(\frac{1}{s^{2}}+\frac{\gamma}{s}+O(1)\right) \quad(s \rightarrow 0)
\end{aligned}
$$

defines a meromorphic function in the region $\operatorname{Re} s>-2$ with a removable singularity at $s=-1$ and a simple pole at $s=0$.

Moving the contour of integration exactly as in [3, Lemma 4.4] we get

$$
\widetilde{S}_{2}^{O}(N)=\operatorname{Res}_{s=0} g_{N}^{O}(s)=h_{N}^{O \prime}(0)+O(N)
$$

where

$$
h_{N}^{O}(s):=\frac{2 N^{s+2}\left(2^{s+2}-2\right)(1+\gamma s)}{(s+1)(s+2)\left(2^{s+2}-1\right) \zeta(s+2)} .
$$

Employing the logarithmic derivative of $h_{N}^{O}$, we get

$$
\widetilde{S}_{2}^{O}(N)=\frac{2 N^{2}}{3 \zeta(2)}\left(\log N+\gamma+\frac{2 \log 2}{3}-\frac{3}{2}-\frac{\zeta^{\prime}(2)}{\zeta(2)}\right)+O(N) .
$$

In similar fashion we find

$$
\widetilde{S}_{2}^{E}(N)=\frac{1}{2 \pi i} \int_{\sigma_{0}-i \infty}^{\sigma_{0}+i \infty} g_{N}^{E}(s) d s=\operatorname{Res}_{s=0} g_{N}^{E}(s)+O(N)=h_{N}^{E}(0)+O(N),
$$

where

$$
\begin{aligned}
& g_{N}^{E}(s)=\frac{2 N^{s+2}}{(s+1)(s+2) 2^{s+1}\left(2^{s+2}-1\right) \zeta(s+2)}\left(\frac{1}{s^{2}}+\frac{\gamma}{s}+O(1)\right) \quad \text { and } \\
& h_{N}^{E}(s)=\frac{2 N^{s+2}(1+\gamma s)}{(s+1)(s+2) 2^{s+1}\left(2^{s+2}-1\right) \zeta(s+2)} .
\end{aligned}
$$

Employing the logarithmic derivative of $h_{N}^{E}$ we get

$$
\widetilde{S}_{2}^{E}(N)=\frac{N^{2}}{6 \zeta(2)}\left(\log N+\gamma-\frac{7 \log 2}{3}-\frac{3}{2}-\frac{\zeta^{\prime}(2)}{\zeta(2)}\right)+O(N) .
$$

Employing $N^{2} S_{0}^{O}(N)-2 N S_{1}^{O}(N)+S_{0}^{O}(N)=\widetilde{S}_{2}^{O}(N)$ and (5.2), (5.4), (5.7), we infer

$$
S_{2}^{O}(N)=\sum_{\substack{m \leq N \\ m \text { odd }}} \frac{\varphi(m)}{m^{2}}=\frac{2}{3 \zeta(2)}\left(\log N+\gamma+\frac{2 \log 2}{3}-\frac{\zeta^{\prime}(2)}{\zeta(2)}\right)+O\left(N^{-1} \log ^{2} N\right),
$$

so for every $\theta>1$,

$$
S_{2}^{O}(N)-S_{2}^{O}\left(\frac{N}{\theta}\right)=\frac{2 \log \theta}{3 \zeta(2)}+O\left(N^{-1} \log ^{2} N\right) .
$$

Employing $N^{2} S_{2}^{E}(N)-2 N S_{1}^{E}(N)+S_{0}^{E}(N)=\widetilde{S}_{2}^{E}(2 N)$ and (5.3), 5.5, ,5.8), we infer

$$
S_{2}^{E}(N)=\sum_{\substack{m \leq N \\ m \text { even }}} \frac{\varphi(2 m)}{m^{2}}=\frac{2}{3 \zeta(2)}\left(\log N+\gamma-\frac{4 \log 2}{3}-\frac{\zeta^{\prime}(2)}{\zeta(2)}\right)+O\left(N^{-1} \log ^{2} N\right),
$$

and so for every $\theta>1$,

$$
S_{2}^{E}(N)-S_{2}^{E}\left(\frac{N}{\theta}\right)=\frac{2 \log \theta}{3 \zeta(2)}+O\left(N^{-1} \log ^{2} N\right)
$$




\section{Distribution of $B$-REDUCED QuAdRAtic irRationals}

In this section we prove a precise asymptotic formula for the cardinality of the set $\mathscr{S}_{B}(\alpha, \beta ; N)$ considered at the end of Section 4. First, we will introduce some number theoretical tools that will play a central role in the proof.

For $\Omega \subset \mathbb{R}^{2}$ and $q, h \geq 1$, denote

$$
\mathscr{N}_{q, h}(\Omega):=\sum_{\substack{(u, v) \in \Omega \\ u v \equiv h}} 1, \quad \mathscr{N}_{q}(\Omega):=\mathscr{N}_{q, 1}(\Omega) .
$$

Using Weil bounds for Kloosterman sums one can show (cf., e.g., [7, Proposition A3]) that, if $(h, q)=1$, then for every $I_{1}, I_{2}$ intervals,

$$
\mathscr{N}_{q, h}\left(I_{1} \times I_{2}\right)=\frac{\varphi(q)}{q^{2}}\left|I_{1}\right|\left|I_{2}\right|+O_{\varepsilon}\left(q^{1 / 2+\varepsilon}\left(1+\frac{\left|I_{1}\right|}{q}\right)\left(1+\frac{\left|I_{2}\right|}{q}\right)\right), \quad \forall \varepsilon>0 .
$$

As long as $(h, q)=1$, the proof of Lemma 2 in [36] still works when replacing \pm 1 by $h$ and employing $(q, m, n h)=(q, m, n)$, and one gets

Lemma 29. Let $q, h$ be integers, $q \geq 2,(h, q)=1$. For every integer $c$ and interval $I$ with $|I|<q$, consider the linear function $f(x)=c \pm x$ such that $f(I) \subseteq[0, q]$. Then

$$
\mathscr{N}_{q, h}(\{(x, y) \mid x \in I, 0 \leq y \leq f(x)\})=\frac{\varphi(q)}{q^{2}} \int_{I} f(x) d x+O_{\varepsilon}\left(q^{1 / 2+\varepsilon}\right), \quad \forall \varepsilon>0 .
$$

First, we estimate $\mathscr{N}_{p,-1}\left(\Omega_{p}^{-}(\alpha, \beta ; N)\right)$, where

$$
\Omega_{p}^{-}(\alpha, \beta ; N):=\left\{(u, v) \mid u \geq \beta p, 0 \leq v \leq \frac{p}{\alpha}, u-v \leq N\right\} .
$$

When $p>\frac{\alpha N}{\alpha \beta-1}$ we have $\beta p>N+\frac{p}{\alpha}$, and so $\Omega_{p}^{-}(\alpha, \beta ; N)=\emptyset$. When $p \leq \frac{\alpha N}{\alpha \beta-1}$ we have $\beta p \leq N+\frac{p}{\alpha}$, giving

$$
\operatorname{Area}\left(\Omega_{p}^{-}(\alpha, \beta ; N)\right)= \begin{cases}(N-\beta p) \frac{p}{\alpha}+\frac{p^{2}}{2 \alpha^{2}} & \text { if } 0<p \leq \frac{N}{\beta} \\ \frac{1}{2}\left(N+\frac{p}{\alpha}-\beta p\right)^{2} & \text { if } \frac{N}{\beta} \leq p \leq \frac{\alpha N}{\alpha \beta-1} .\end{cases}
$$

When $p \leq \frac{N}{\beta}$, apply estimate 6.1 with $I_{1} \times I_{2}=[\beta p, N] \times\left[0, \frac{p}{\alpha}\right]$, and Lemma 29 with $f(x)=$ $x-N$ and $I=\left[N, N+\frac{p}{\alpha}\right]$, of length $\frac{p}{\alpha} \leq p$, together with $\left[6.3\right.$. For the case $\frac{N}{\beta} \leq p \leq \frac{\alpha N}{\alpha \beta-1}$, apply estimate 6.1 with $I_{1} \times I_{2}=\left[\beta p, N+\frac{p}{\alpha}\right] \times\left[0, \frac{p}{\alpha}\right]$ and Lemma 29 with $f(x)=x-N$ and $I=\left[\beta p, N+\frac{p}{\alpha}\right]$, of length $\leq \frac{p}{\alpha}$, together with $[6.3)$, to get

$$
\begin{aligned}
& \left|\mathscr{S}_{B}(\alpha, \beta ; N)\right|=\sum_{1 \leq p \leq \frac{\alpha N}{\alpha \beta-1}} \mathscr{N}_{p,-1}\left(\Omega_{p}^{-}(\alpha, \beta ; N)\right) \\
& =\sum_{1 \leq p \leq \frac{N}{\beta}} \frac{\varphi(p)}{p^{2}}\left((N-\beta p) \frac{p}{\alpha}+\frac{p^{2}}{2 \alpha^{2}}\right)+\sum_{\frac{N}{\beta}<p \leq \frac{\alpha N}{\alpha \beta-1}} \frac{\varphi(p)}{2 p^{2}}\left(N+\frac{p}{\alpha}-\beta p\right)^{2}+O_{\varepsilon}\left(N^{3 / 2+\varepsilon}\right) \\
& =\frac{N}{\alpha} S_{1}\left(\frac{N}{\beta}\right)-\frac{\beta}{\alpha} S_{0}\left(\frac{N}{\beta}\right)+\frac{1}{2 \alpha^{2}} S_{0}\left(\frac{N}{\beta}\right)+\frac{N^{2}}{2}\left(S_{2}\left(\frac{\alpha N}{\alpha \beta-1}\right)-S_{2}\left(\frac{N}{\beta}\right)\right) \\
& \quad+\frac{(1-\alpha \beta)^{2}}{2 \alpha^{2}}\left(S_{0}\left(\frac{\alpha N}{\alpha \beta-1}\right)-S_{0}\left(\frac{N}{\beta}\right)\right) \\
& \quad+\frac{(1-\alpha \beta) N}{\alpha}\left(S_{1}\left(\frac{\alpha N}{\alpha \beta-1}\right)-S_{1}\left(\frac{N}{\beta}\right)\right)+O_{\varepsilon}\left(N^{3 / 2+\varepsilon}\right) .
\end{aligned}
$$


Combining (6.4) with (5.1) we infer

$$
\begin{aligned}
\left|\mathscr{S}_{B}(\alpha, \beta ; N)\right| & =\frac{N^{2}}{2 \zeta(2)} \log \left(\frac{\alpha \beta}{\alpha \beta-1}\right)+O_{\varepsilon}\left(N^{3 / 2+\varepsilon}\right) \\
& =\frac{N^{2}}{2 \zeta(2)} \iint_{[\alpha, \infty) \times\left[0, \frac{1}{\beta}\right]} \frac{d u d v}{(u-v)^{2}}+O_{\varepsilon}\left(N^{3 / 2+\varepsilon}\right) .
\end{aligned}
$$

Theorem 4 follows from $\left(6.5\right.$ and the approximation argument: ${ }^{2}$ in Section 8 , taking $N=$ $e^{R / 2}$. Corollary 5 follows taking $\beta=1$.

\section{Estimating the Cardinality of the $\operatorname{Sets} \mathscr{S}_{ \pm}(\alpha, \beta ; N)$}

To the end of parameterizing $\mathscr{S}(\alpha, \beta ; N)$ defined in Subsection 2.3 , write

$$
\mathscr{S}(\alpha, \beta ; N)=\mathscr{S}_{+}(\alpha, \beta ; N) \cup \mathscr{S}_{-}(\alpha, \beta ; N),
$$

where $\mathscr{S}_{i}(\alpha, \beta ; N)=\mathscr{S}(\alpha, \beta ; N) \cap\{e=i\}, i= \pm 1$, are disjoint sets.

We start by proving the asymptotic formula

$$
\left|\mathscr{S}_{+}(\alpha, \beta ; N)\right|=\frac{N^{2}}{6 \zeta(2)} \log \left(\frac{\alpha \beta+1}{\alpha \beta}\right)+O_{\varepsilon}\left(N^{3 / 2+\varepsilon}\right) .
$$

For every $N \geq 2, \alpha, \beta \geq 1$ consider the sets

$$
\begin{aligned}
& \mathscr{A}_{1}(\alpha, \beta ; N):=\left\{(m, u, v) \mid \begin{array}{c}
u \geq \beta m, 0 \leq v \leq \frac{m}{\beta}, u+v \leq N \\
m \text { odd }, u v \equiv 1 \quad(\bmod m), u, v \text { even }
\end{array}\right\}, \\
& \mathscr{A}_{2}(\alpha, \beta ; N):=\left\{(m, u, v) \mid \begin{array}{c}
u \geq \beta m, 0 \leq v \leq \frac{m}{\alpha}, u+v \leq N \\
m \text { even, } u v \equiv 1 \quad(\bmod 2 m), u, v \text { odd }
\end{array}\right\} .
\end{aligned}
$$

The last condition in the definition of $\mathscr{A}_{2}(\alpha, \beta ; N)$ is obsolete as $u v \equiv 1(\bmod 2 m)$ implies $u, v$ odd.

Lemma 30. The map

$$
\Phi: \mathscr{S}_{+}(\alpha, \beta ; N) \rightarrow \mathscr{A}_{1}(\alpha, \beta ; N) \cup \mathscr{A}_{2}(\alpha, \beta ; N), \quad \Phi\left(\begin{array}{cc}
p^{\prime} & p \\
q^{\prime} & q
\end{array}\right):=\left(p, p^{\prime}, q\right)
$$

is a bijection.

Proof. The map $\Phi$ is well defined because $\sigma=\left(\begin{array}{c}p^{\prime} \\ q^{\prime} \\ q\end{array}\right) \equiv I_{2}$ or $J_{2}(\bmod 2)$ entails $p$ odd $\Longrightarrow p^{\prime}, q$ even and $p$ even $\Longrightarrow p^{\prime}, q$ odd. We show that for every $(m, u, v) \in \mathscr{A}_{1}(\alpha, \beta ; N) \cup \mathscr{A}_{2}(\alpha, \beta ; N)$,

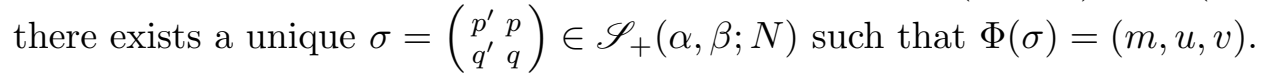

Suppose first $(m, u, v)=\left(p, p^{\prime}, q\right) \in \mathscr{A}_{1}(\alpha, \beta ; N)$. We must have $p^{\prime} q-p q^{\prime}=1$, or, equivalently, $q^{\prime}:=\frac{u v-1}{p}$. Since $u v-1$ is odd, we have that $q^{\prime}$ odd, so $\sigma \equiv J_{2}(\bmod 2)$.

When $(m, u, v)=\left(p, p^{\prime}, q\right) \in \mathscr{A}_{2}(\alpha, \beta ; N)$, we similarly have $q^{\prime}:=\frac{u v-1}{p}$. The condition $u v \equiv 1$ $(\bmod 2 p)$ gives $\sigma \equiv I_{2}(\bmod 2)$.

In both situations we have $v<m<u$, so $v=q \leq \frac{u v-1}{m}=q^{\prime}<u=p^{\prime}$.

\footnotetext{
${ }^{2}$ For the purpose of this approximation $e_{i}=-1$ for all $i$. Also, the $a_{i}$ 's are not necessarily even, but only $a_{i} \geq 2$ is needed.
} 
As a corollary we obtain

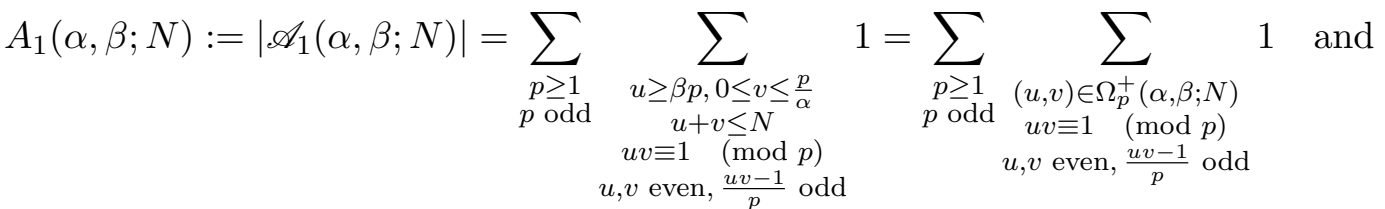

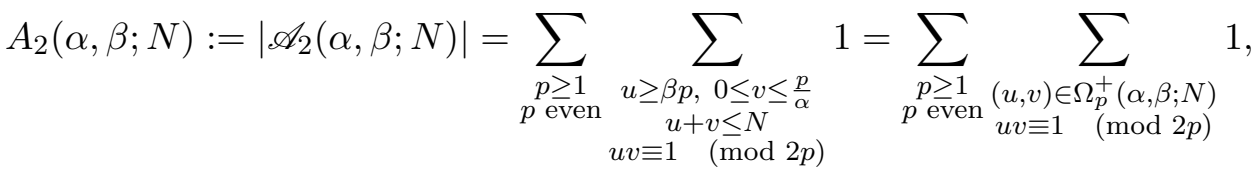

where we consider the region

$$
\Omega_{p}^{+}(\alpha, \beta ; N):=\left\{(u, v) \mid u \geq \beta p, 0 \leq v \leq \frac{p}{\alpha}, u+v \leq N\right\} .
$$

When $p>\frac{N}{\beta}$ we have $\Omega_{p}^{+}(\alpha, \beta ; N)=\emptyset$. When $p \leq \frac{N}{\beta}$ we have

$$
\operatorname{Area}\left(\Omega_{p}^{+}(\alpha, \beta ; N)\right)= \begin{cases}(N-\beta p) \frac{p}{\alpha}-\frac{p^{2}}{2 \alpha^{2}} & \text { if } 0 \leq p \leq \frac{\alpha N}{\alpha \beta+1} \\ \frac{(N-\beta p)^{2}}{2} & \text { if } \frac{\alpha N}{\alpha \beta+1} \leq p \leq \frac{N}{\beta}\end{cases}
$$

Writing $u=2 a, v=2 b$, we have that $(u, v) \in \Omega_{p}^{+}(\alpha, \beta ; N) \Longleftrightarrow(a, b) \in \frac{1}{2} \Omega_{p}^{+}(\alpha, \beta ; N)$, and that $\frac{u v-1}{p}=\frac{4 a b-1}{p}$ is odd $\Longleftrightarrow 4 a b-1 \equiv p(\bmod 2 p) \Longleftrightarrow 4 a b \equiv p+1(\bmod 2 p) \Longleftrightarrow 2 a b \equiv \frac{p+1}{2}$ $(\bmod p) \Longleftrightarrow a b \equiv \overline{2} \cdot \frac{p+1}{2}(\bmod p)$, where $\overline{2} \cdot 2 \equiv 1(\bmod p)$, so that

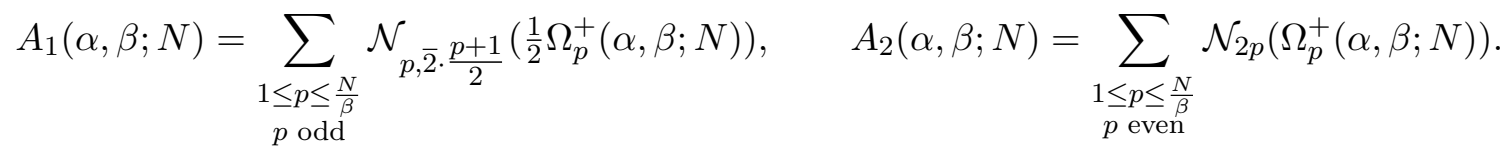

First, we estimate $A_{1}(\alpha, \beta ; N)$. Here $p$ is odd, $\left(\frac{p+1}{2}, p\right)=1,(\overline{2}, p)=1$, so $\left(\overline{2} \cdot \frac{p+1}{2}, p\right)=1$. When $p \leq \frac{\alpha N}{\alpha \beta+1}$, we apply estimate 6.1 with $I_{1} \times I_{2}=\left[\beta p, N-\frac{p}{\alpha}\right] \times\left[0, \frac{p}{\alpha}\right]$ and Lemma 29 with $f(x)=N-x$ and $I=\left[N-\frac{p}{\alpha}, N\right]$, of length $\frac{p}{\alpha} \leq p$, together with (7.2). For the case $\frac{\alpha N}{\alpha \beta+1} \leq p \leq \frac{N}{\beta}$, we apply Lemma 29 with $f(x)=N-x$ and $I=[\beta p, N]$, of length $\leq \frac{p}{\alpha}$, together with $(7.2)$, to get

$$
\begin{aligned}
& A_{1}(\alpha, \beta ; N)=\frac{1}{4} \sum_{\substack{1 \leq p \leq \frac{N}{\beta} \\
p \text { odd }}} \frac{\varphi(p)}{p^{2}} \operatorname{Area}\left(\Omega_{p}^{+}(\alpha, \beta ; N)\right) \\
& =\frac{1}{4} \sum_{\substack{1 \leq p \leq \frac{\alpha N}{\alpha \beta+1} \\
p \text { odd }}} \frac{\varphi(p)}{p^{2}}\left((N-\beta p) \frac{p}{\alpha}-\frac{p^{2}}{2 \alpha^{2}}\right)+\frac{1}{4} \sum_{\substack{\alpha N \\
\frac{\alpha N}{\alpha \beta+1} \leq \leq \leq N \\
p \text { odd }}} \frac{\varphi(p)}{p^{2}} \cdot \frac{(N-\beta p)^{2}}{2}+O_{\varepsilon}\left(N^{3 / 2+\varepsilon}\right) \\
& =\frac{N}{4 \alpha} S_{1}^{O}\left(\frac{\alpha N}{\alpha \beta+1}\right)-\frac{\beta}{4 \alpha} S_{0}^{O}\left(\frac{\alpha N}{\alpha \beta+1}\right)-\frac{1}{8 \alpha^{2}} S_{0}^{O}\left(\frac{\alpha N}{\alpha \beta+1}\right) \\
& \quad+\frac{N^{2}}{8}\left(S_{2}^{O}\left(\frac{N}{\beta}\right)-S_{2}^{O}\left(\frac{\alpha N}{\alpha \beta+1}\right)\right)-\frac{\beta N}{4}\left(S_{1}^{O}\left(\frac{N}{\beta}\right)-S_{1}^{O}\left(\frac{\alpha N}{\alpha \beta+1}\right)\right) \\
& \quad+\frac{\beta^{2}}{8}\left(S_{0}^{O}\left(\frac{N}{\beta}\right)-S_{0}^{O}\left(\frac{\alpha N}{\alpha \beta+1}\right)\right)+O_{\varepsilon}\left(N^{3 / 2+\varepsilon}\right) .
\end{aligned}
$$


Combining (7.3) with (5.2), (5.3) and (5.9), we infer after a short calculation

$$
A_{1}(\alpha, \beta)=\frac{N^{2}}{12 \zeta(2)} \log \left(\frac{\alpha \beta+1}{\alpha \beta}\right)+O_{\varepsilon}\left(N^{3 / 2+\varepsilon}\right) .
$$

Next, we estimate $A_{2}(\alpha, \beta ; N)$. In this case we have

$$
\begin{aligned}
& A_{2}(\alpha, \beta ; N)=\frac{1}{4} \sum_{\substack{1 \leq p \leq \frac{N}{\beta} \\
p \text { even }}} \frac{\varphi(2 p)}{p^{2}} \operatorname{Area}\left(\Omega_{p}^{+}(\alpha, \beta ; N)\right) \\
& =\frac{1}{4} \sum_{\substack{1 \leq p \leq \frac{\alpha N}{\alpha \beta+1} \\
p \text { even }}} \frac{\varphi(2 p)}{p^{2}}\left((N-\beta p) \frac{p}{\alpha}-\frac{p^{2}}{2 \alpha^{2}}\right)+\frac{1}{4} \sum_{\substack{\alpha N \\
\frac{\alpha \beta}{\alpha+1} \leq p \leq \frac{N}{\beta}}} \frac{\varphi(2 p)}{p^{2}} \cdot \frac{(N-\beta p)^{2}}{2}+O_{\varepsilon}\left(N^{3 / 2+\varepsilon}\right) \\
& =\frac{N}{4 \alpha} S_{1}^{E}\left(\frac{\alpha N}{\alpha \beta+1}\right)-\frac{\beta}{4 \alpha} S_{0}^{E}\left(\frac{\alpha N}{\alpha \beta+1}\right)-\frac{1}{8 \alpha^{2}} S_{0}^{E}\left(\frac{\alpha N}{\alpha \beta+1}\right) \\
& \quad+\frac{N^{2}}{8}\left(S_{2}^{E}\left(\frac{N}{\beta}\right)-S_{2}^{E}\left(\frac{\alpha N}{\alpha \beta+1}\right)\right)-\frac{\beta N}{4}\left(S_{1}^{E}\left(\frac{N}{\beta}\right)-S_{1}^{E}\left(\frac{\alpha N}{\alpha \beta+1}\right)\right) \\
& \quad+\frac{\beta^{2}}{8}\left(S_{0}^{E}\left(\frac{N}{\beta}\right)-S_{0}^{E}\left(\frac{\alpha N}{\alpha \beta+1}\right)\right)+O_{\varepsilon}\left(N^{3 / 2+\varepsilon}\right) .
\end{aligned}
$$

Combining this with (5.3), (5.5) and (5.10), we infer

$$
A_{2}(\alpha, \beta)=\frac{N^{2}}{12 \zeta(2)} \log \left(\frac{\alpha \beta+1}{\alpha \beta}\right)+O_{\varepsilon}\left(N^{3 / 2+\varepsilon}\right) .
$$

The estimate (7.1) follows from (7.4) and 7.5).

To estimate $\left|\mathscr{S}_{-}(\alpha, \beta ; N)\right|$, consider the region $\Omega_{p}^{-}(\alpha, \beta ; N)$ defined by 6.2$)$ and employ $p^{\prime} q-$ $p q^{\prime}=-1$, which gives $p^{\prime} q \equiv-1(\bmod p)$. We first observe as above that

$$
\begin{aligned}
& \left|\mathscr{S}_{-}(\alpha, \beta ; N)\right|=\sum_{\substack{1 \leq p \leq \frac{\alpha N}{\alpha \beta-1} \\
p \text { odd }}} \mathscr{N}_{p, \overline{2} \cdot \frac{p-1}{2}}\left(\frac{1}{2} \Omega_{p}^{-}(\alpha, \beta ; N)\right)+\sum_{\substack{1 \leq p \leq \frac{\alpha N}{\alpha \beta-1} \\
p \text { even }}} \mathscr{N}_{2 p,-1}\left(\Omega_{p}^{-}(\alpha, \beta ; N)\right) \\
& =: B_{1}(\alpha, \beta ; N)+B_{2}(\alpha, \beta ; N) \text {. }
\end{aligned}
$$

Proceeding exactly as in Section 6 and as in the estimation for $A_{1}(\alpha, \beta ; N)$ and $A_{2}(\alpha, \beta ; N)$ above, the only difference being $u v \equiv-1(\bmod p)$ in place of $u v \equiv 1(\bmod p)$, we show that

$$
\begin{aligned}
& B_{1}(\alpha, \beta ; N)=\frac{N^{2}}{12 \zeta(2)} \log \left(\frac{\alpha \beta}{\alpha \beta-1}\right)+O_{\varepsilon}\left(N^{3 / 2+\varepsilon}\right), \\
& B_{2}(\alpha, \beta ; N)=\frac{N^{2}}{12 \zeta(2)} \log \left(\frac{\alpha \beta}{\alpha \beta-1}\right)+O_{\varepsilon}\left(N^{3 / 2+\varepsilon}\right),
\end{aligned}
$$

and therefore

$$
\left|\mathscr{S}_{-}(\alpha, \beta ; N)\right|=\frac{N^{2}}{6 \zeta(2)} \log \left(\frac{\alpha \beta}{\alpha \beta-1}\right)+O_{\varepsilon}\left(N^{3 / 2+\varepsilon}\right) .
$$

Combining (7.1) and (7.6), we get

$$
\left|\mathscr{S}_{-}\left(\alpha, \beta_{1} ; N\right)\right|+\left|\mathscr{S}_{+}\left(\alpha, \beta_{2} ; N\right)\right|=C\left(\alpha, \beta_{1}, \beta_{2}\right) N^{2}+O_{\varepsilon}\left(N^{3 / 2+\varepsilon}\right),
$$

with $C\left(\alpha, \beta_{1}, \beta_{2}\right)$ as in Theorem 1 . 


\section{Distribution of ECF-REDUCEd QUAdRAtiC IRRATionals}

This section completes the proofs of Theorems 1 and 4 through a careful analysis of the error resulted while approximating $r_{E}\left(\alpha, \beta_{1}, \beta_{2} ; R\right)$ by $\left|\mathscr{S}_{-}\left(\alpha, \beta_{1} ; N\right)\right|+\left|\mathscr{S}_{+}\left(\alpha, \beta_{2} ; N\right)\right|$ when $N=e^{R / 2} \rightarrow \infty$.

First, we show that the error resulting from replacing the spectral radius of $\widetilde{\Omega}_{E}(\omega)$ by the trace is negligible.

Lemma 31. $\mathfrak{r}\left(\widetilde{\Omega}_{E}(\omega)^{k}\right)<\operatorname{Tr}\left(\widetilde{\Omega}_{E}(\omega)^{k}\right) \leq \mathfrak{r}\left(\widetilde{\Omega}_{E}(\omega)^{k}\right)+\frac{1}{2}, \quad \forall \omega \in \mathscr{R}_{E}, \forall k \geq 1$.

Proof. First, we show $\eta:=\mathfrak{r}\left(\widetilde{\Omega}_{E}(\omega)\right) \geq 2$. We have $\mathfrak{r}\left(\Omega_{E}(\omega)\right)=q_{n} \omega+q_{n-1} e_{n} \geq q_{n}-q_{n-1}$ and

$$
q_{n}-q_{n-1} \geq q_{n-1}-q_{n-2} \geq \cdots \geq q_{1}-q_{0}=1 .
$$

At least one of the inequalities above is strict, or else $e_{1}=\cdots=e_{n}=-1$ and $a_{1}=\cdots=a_{n}=2$, giving $\omega=[\overline{(2,-1)}]=1$, contradiction. We infer $\mathfrak{r}\left(\Omega_{E}(\omega)\right) \geq 2$. The inequality $\eta \geq 2$ follows replacing $n$ by $2 n$ when $\delta_{n}=-1$. This leads to $\eta^{k}<\operatorname{Tr}\left(\widetilde{\Omega}_{E}(\omega)^{k}\right)=\eta^{k}+\eta^{-k} \leq \eta^{k}+\eta^{-1} \leq$ $\eta^{k}+\frac{1}{2}$.

Corollary 32. $\mathscr{T}_{k}(N)=\emptyset$ whenever $k>\log _{2} N$.

Proof. Let $(\omega, k) \in \mathscr{T}_{k}(N)$. We have $\eta^{k}<\eta^{k}+\eta^{-k}=\operatorname{Tr}\left(\widetilde{\Omega}_{E}(\omega)^{k}\right) \leq N$, giving $k \leq \frac{\log N}{\log \eta} \leq$ $\frac{\log N}{\log 2}=\log _{2} N$.

Denote

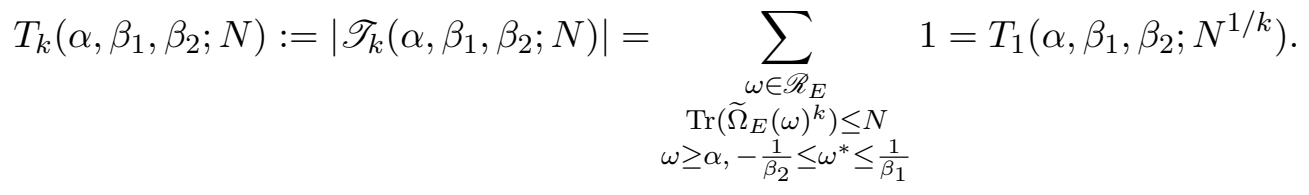

Upon Lemma 31 we have, with $r_{E}\left(\alpha, \beta_{1}, \beta_{2} ; R\right)$ as in 1.9 and $N=e^{R / 2}$,

$$
T_{1}\left(\alpha, \beta_{1}, \beta_{2} ; N-\frac{1}{2}\right) \leq r_{E}\left(\alpha, \beta_{1}, \beta_{2} ; R\right) \leq T_{1}\left(\alpha, \beta_{1}, \beta_{2} ; N\right) .
$$

Assume $\alpha, \beta_{1}, \beta_{1} \geq 1$ with $\alpha \beta_{1}>1$. Consider

$$
S\left(\alpha, \beta_{1}, \beta_{2} ; N\right):=\sum_{k=1}^{\infty} T_{k}\left(\alpha, \beta_{1}, \beta_{2} ; N\right) .
$$

By Corollary 20 we can writ€ ${ }^{3}$

$$
S\left(\alpha, \beta_{1}, \beta_{2} ; N\right)=\left|\mathscr{W}_{E}^{+}\left(\alpha, \beta_{1}, \beta_{2} ; N\right)\right|=S_{-}\left(\alpha, \beta_{1} ; N\right)+S_{+}\left(\alpha, \beta_{2} ; N\right),
$$

with $S_{-}\left(\alpha, \beta_{1} ; N\right)$ collecting the contribution of terms with $\omega^{*} \in\left(0, \frac{1}{\beta_{1}}\right]$ and $S_{+}\left(\alpha, \beta_{2} ; N\right)$ collecting the contribution of terms with $\omega^{*} \in\left[-\frac{1}{\beta_{2}}, 0\right)$.

By Corollary 32 and $T_{k}\left(\alpha, \beta_{1}, \beta_{2} ; N\right) \ll N^{2 / k}$ we infer 4

$$
\begin{aligned}
S\left(\alpha, \beta_{1}, \beta_{2} ; N\right) & =T_{1}\left(\alpha, \beta_{1}, \beta_{2} ; N\right)+O\left(\sum_{2 \leq k \leq \log _{2} N} N^{2 / k}\right) \\
& =T_{1}\left(\alpha, \beta_{1}, \beta_{2} ; N\right)+O(N \log N) .
\end{aligned}
$$

\footnotetext{
${ }^{3}$ Recall that $\left(\left(a_{1}, e_{1}\right), \ldots,\left(a_{n}, e_{n}\right)\right) \in \mathscr{W}_{E}^{+}$and $\omega:=\left[\overline{\left(a_{1}, e_{1}\right), \ldots,\left(a_{n}, e_{n}\right)}\right]$ imply $\widetilde{\Omega}_{E}(\omega)=\Omega_{E}(\omega)$.

${ }^{4}$ Note that the set $\mathscr{S}_{+}(1,1 ; N)$ is finite, but $\mathscr{S}_{-}(1,1 ; N)$ and $\mathscr{S}_{B}(1,1 ; N)$ may be a priori infinite.
} 
Lemma 33. (i) $\left|\left\{\sigma=\left(\begin{array}{cc}p^{\prime} & e p \\ q^{\prime} & e q\end{array}\right) \in \mathscr{S}_{+} \mid q\left(q^{\prime}+e q\right) \leq N, p^{\prime} \leq A N\right\}\right|=O_{A, \varepsilon}\left(N^{3 / 2+\varepsilon}\right)$.

(ii) $\left|\left\{\sigma=\left(\begin{array}{c}p^{\prime}-p \\ q^{\prime}-q\end{array}\right) \in \mathscr{S}_{+} \mid p(p-q) \leq N, p^{\prime} \leq A N\right\}\right|=O_{A, \varepsilon}\left(N^{3 / 2+\varepsilon}\right)$.

Proof. (i) When $e=+1$ we get $q \leq \sqrt{N}$ and $p q^{\prime}=p^{\prime} q-1<A N^{3 / 2}$. Fix $p^{\prime}$ and $q$. The number of admissible values for $p$ is at most the number of divisors of $p^{\prime} q-1$, so it is $O_{A, \varepsilon}\left(N^{\varepsilon}\right)$. Hence the number of $\sigma$ 's is $O_{A, \varepsilon}\left(N^{3 / 2+\varepsilon}\right)$.

When $e=-1$ we consider two cases:

(i $\left.i_{1}\right) q \leq \sqrt{N}$. Fixing $p^{\prime}$ and $q$, the number of admissible values for $p$ is at most the number of divisors of $p^{\prime} q+1$, so it is again $O_{A, \varepsilon}\left(N^{\varepsilon}\right)$.

$\left(\mathrm{i}_{2}\right) q \geq \sqrt{N}$. In this case $0<k:=q^{\prime}-q \leq \frac{N}{q} \leq \sqrt{N}$ and $\left(p^{\prime}-p\right) q=p k-1$. Fixing $p$ and $k$, the number of admissible values for $q$ is $O_{A, \varepsilon}\left(N^{\varepsilon}\right)$ as above, and $p, k$ and $q$ completely determine $\sigma$.

(ii) The proof is similar. Consider first $p \leq \sqrt{N}$ and fix $p$ and $q^{\prime}$, which limits the number of admissible values for $p^{\prime}$ to $O_{A, \varepsilon}\left(N^{\varepsilon}\right)$. In the second case $p \geq \sqrt{N}$ gives $0<\ell:=p-q \leq \frac{N}{p} \leq \sqrt{N}$. We proceed as in $\left(\mathrm{i}_{2}\right)$. Fix $\ell$ and $q^{\prime}$ and observe that the equality $q\left(p^{\prime}-q^{\prime}\right)+1=\ell q^{\prime}$ limits the number of admissible values for $q$ to $O_{A, \varepsilon}\left(N^{\varepsilon}\right)$.

Employing equality $(2.5)$ and $T_{E}^{n}(\omega)=\omega$, we get

$$
\left|\omega-\frac{p_{n-1}}{q_{n-1}}\right|=\left|\frac{e_{n} p_{n-1}+\omega p_{n}}{e_{n} q_{n-1}+\omega q_{n}}-\frac{p_{n-1}}{q_{n-1}}\right|=\left|\frac{e_{n}}{q_{n-1}\left(q_{n}+\frac{e_{n}}{\omega} q_{n-1}\right)}\right| \leq \frac{1}{q_{n-1}\left(q_{n}-q_{n-1}\right)} .
$$

Employing equality (2.7), we get

$$
\left|-\frac{1}{\omega^{*}}-\frac{e_{n} p_{n}}{p_{n-1}}\right|=\left|\frac{\omega^{*} e_{n} q_{n}-e_{n} p_{n}}{\omega^{*} q_{n-1}-p_{n-1}}-\frac{e_{n} p_{n}}{p_{n-1}}\right|=\left|\frac{\omega^{*}}{p_{n-1}\left(p_{n-1}-\omega^{*} q_{n-1}\right)}\right| \leq \frac{1}{p_{n-1}\left(p_{n-1}-q_{n-1}\right)} .
$$

Furthermore, we have $\omega>\frac{p_{n-1}}{q_{n-1}}$ when $e_{n}=+1$ and $\omega<\frac{p_{n-1}}{q_{n-1}}$ when $e_{n}=-1$. When $e_{n}=+1$ we also have $-\frac{1}{\omega^{*}}>\frac{p_{n}}{p_{n-1}}$, so $\frac{p_{n}}{p_{n-1}} \geq \beta_{2} \Longrightarrow 0>-\omega^{*} \geq-\frac{1}{\beta_{2}}$, while when $e_{n}=-1$ we have $\frac{1}{\omega^{*}}>\frac{p_{n}}{p_{n-1}}$, so $\frac{p_{n}}{p_{n-1}} \geq \beta_{1} \Longrightarrow 0<\omega^{*} \leq \frac{1}{\beta_{1}}$.

Lemma 33 will be applied with $A=1$ (making the error term independent of $\beta_{2}$ ) for $e=+1$, and with $A=1+\frac{\alpha}{\alpha \beta_{1}-1}$ when $e=-1$.

Combining Lemma 33 and (8.2), (8.4), 8.5), 7.7), we infer

$$
\begin{aligned}
S\left(\alpha, \beta_{1}, \beta_{2} ; N\right) & \sum_{\substack{\sigma \in \mathscr{S}_{-}\left(\alpha, \beta_{1}-\frac{1}{N} ; N\right) \cup \mathscr{S}_{+}\left(\alpha-\frac{1}{N}, \beta_{2}-\frac{1}{N} ; N\right) \\
\min \left\{q\left(q^{\prime}-q\right), p(p-q)\right\} \geq N}} 1+O_{\alpha, \beta_{1}, \varepsilon}\left(N^{3 / 2+\varepsilon}\right) \\
& \leq\left|\mathscr{S}_{-}\left(\alpha, \beta_{1}-\frac{1}{N} ; N\right)\right|+\left|\mathscr{S}_{+}\left(\alpha-\frac{1}{N}, \beta_{2}-\frac{1}{N} ; N\right)\right|+O_{\alpha, \beta_{1}, \varepsilon}\left(N^{3 / 2+\varepsilon}\right) \\
& =C\left(\alpha, \beta_{1}, \beta_{2}\right) N^{2}+O_{\alpha, \beta_{1}, \varepsilon}\left(N^{3 / 2+\varepsilon}\right),
\end{aligned}
$$

and also

$$
\begin{aligned}
S\left(\alpha, \beta_{1}, \beta_{2} ; N\right) & \geq \sum_{\substack{\sigma \in \mathscr{S}_{-}\left(\alpha+\frac{1}{N}, \beta_{1} ; N\right) \cup \mathscr{S}_{+}\left(\alpha, \beta_{2} ; N\right) \\
\min \left\{q\left(q^{\prime}-q\right), p(p-q)\right\} \geq N}} 1 \\
& =\left|\mathscr{S}_{-}\left(\alpha+\frac{1}{N}, \beta_{1} ; N\right)\right|+\left|\mathscr{S}_{+}\left(\alpha, \beta_{2} ; N\right)\right|+O_{\alpha, \beta_{1}, \varepsilon}\left(N^{3 / 2+\varepsilon}\right) \\
& =C\left(\alpha, \beta_{1}, \beta_{2}\right) N^{2}+O_{\alpha, \beta_{1}, \varepsilon}\left(N^{3 / 2+\varepsilon}\right) .
\end{aligned}
$$


Taking $N=e^{R / 2}$ and combining (8.1), (8.3), (8.6) and (8.7), we infer

$$
r_{E}\left(\alpha, \beta_{1}, \beta_{2} ; R\right)=C\left(\alpha, \beta_{1}, \beta_{2}\right) e^{R}+O_{\alpha, \beta_{1}, \varepsilon}\left(e^{(3 / 4+\varepsilon) R}\right) .
$$

This proves Theorem 1 .

The analysis of the error resulting while approximating $r_{B}(\alpha, \beta ; R)$ by $\left|\mathscr{S}_{B}(\alpha, \beta ; N)\right|$ is similar. In this case all $e_{i}$ 's are equal to -1 . This completes the proof of Theorem 4

\section{APPENDIX}

To illustrate the difference between $E$-reduced QIs, $B$-reduced QIs and reduced QIs, we consider a few examples. We set $a=2 k, a_{1}=2 k_{1}, a_{2}=2 k_{2}$, with $k, k_{1}, k_{2} \in \mathbb{N}$.

Here we denote

$$
\left[b_{1}, b_{2}, b_{3}, \ldots\right]:=b_{1}+\frac{1}{b_{2}+\frac{1}{b_{3}+\ldots}}, \quad b_{i} \in \mathbb{N} .
$$

We say that the QI $\omega>1$ is (regular) reduced if $\omega^{*} \in(-1,0)$. It is well-known that this is equivalent with $\omega=\left[\overline{b_{1}, \ldots, b_{d}}\right]$ for some $d \geq 1$ and $b_{1}, \ldots, b_{d} \in \mathbb{N}$.

Example 1. $\omega=[\overline{(a,-1)}]=a-\frac{1}{\omega}$ has minimal polynomial $X^{2}-2 k X+1$ and $\operatorname{disc}(\omega)=4\left(k^{2}-1\right)$.

The $E$-reduced QI $\omega=k+\sqrt{k^{2}-1}>1$ is not reduced as $\omega^{*}=k-\sqrt{k^{2}-1} \in[0,1]$.

The largest eigenvalue of $\Omega_{E}(\omega)=\left(\begin{array}{cc}a & -1 \\ 1 & 0\end{array}\right)$ is $\mathfrak{r}\left(\Omega_{E}(\omega)\right)=k+\sqrt{k^{2}-1}$. Here $\widetilde{\Omega}_{E}(\omega)=\Omega_{E}(\omega)^{2}$ and so $\varrho_{E}(\omega)=4 \log \left(k+\sqrt{k^{2}-1}\right)$.

Example 2. $\omega=\left[\overline{\left(a_{1}, 1\right),\left(a_{2},-1\right)}\right]=a_{1}+\frac{1}{a_{2}-\frac{1}{\omega}}$ has minimal polynomial $k_{2} X^{2}-\left(1+2 k_{1} k_{2}\right) X+$ $k_{1}$ and $\operatorname{disc}(\omega)=4 k_{1}^{2} k_{2}^{2}+1$.

The $E$-reduced QI $\omega=\frac{2 k_{1} k_{2}+1+\sqrt{4 k_{1}^{2} k_{2}^{2}+1}}{2 k_{2}}$ is not reduced as $\omega^{*}=\frac{2 k_{1}}{2 k_{1} k_{2}+1+\sqrt{4 k_{1}^{2} k_{2}^{2}+1}} \in(0,1)$.

The largest eigenvalue of $\Omega_{E}(\omega)=\left(\begin{array}{cc}a_{1} & 1 \\ 1 & 0\end{array}\right)\left(\begin{array}{cc}a_{2} & -1 \\ 1 & 0\end{array}\right)=\left(\begin{array}{cc}a_{1} a_{2}+1 & -a_{1} \\ a_{2} & -1\end{array}\right)$ is $\mathfrak{r}\left(\Omega_{E}(\omega)\right)=2 k_{1} k_{2}+$ $\sqrt{4 k_{1}^{2} k_{2}^{2}+1}$. Here $\widetilde{\Omega}_{E}(\omega)=\Omega_{E}(\omega)^{2}$ and so $\varrho_{E}(\omega)=4 \log \left(2 k_{1} k_{2}+\sqrt{4 k_{1}^{2} k_{2}^{2}+1}\right)$.

Example 3. $\omega=\left[\overline{\left(a_{1},-1\right),\left(a_{2}, 1\right)}\right]=a_{1}-\frac{1}{a_{2}+\frac{1}{\omega}}$ has minimal polynomial $k_{2} X^{2}+\left(1-2 k_{1} k_{2}\right) X-$ $k_{1}$ and $\operatorname{disc}(\omega)=4 k_{1}^{2} k_{2}^{2}+1$.

The $E$-reduced QI $\omega=\frac{2 k_{1} k_{2}-1+\sqrt{4 k_{1}^{2} k_{2}^{2}+1}}{2 k_{2}}$ is also reduced as $\omega^{*}=\frac{-2 k_{1}}{2 k_{1} k_{2}-1+\sqrt{4 k_{1}^{2} k_{2}^{2}+1}} \in(-1,0)$.

The largest eigenvalue of $\Omega_{E}(\omega)=\left(\begin{array}{cc}a_{1} & -1 \\ 1 & 0\end{array}\right)\left(\begin{array}{cc}a_{2} & 1 \\ 1 & 0\end{array}\right)=\left(\begin{array}{cc}a_{1} a_{2}-1 & a_{1} \\ a_{2} & 1\end{array}\right)$ is $\mathfrak{r}\left(\Omega_{E}(\omega)\right)=2 k_{1} k_{2}+$ $\sqrt{4 k_{1}^{2} k_{2}^{2}+1}$. Here $\widetilde{\Omega}_{E}(\omega)=\Omega_{E}(\omega)^{2}$ and so $\varrho_{E}(\omega)=4 \log \left(2 k_{1} k_{2}+\sqrt{4 k_{1}^{2} k_{2}^{2}+1}\right)$.

Finally notice that the equality

$$
\left(\begin{array}{cc}
a_{1} & -1 \\
1 & 0
\end{array}\right)\left(\begin{array}{cc}
a_{2} & 1 \\
1 & 0
\end{array}\right)=\left(\begin{array}{cc}
a_{1}-1 & 1 \\
1 & 0
\end{array}\right)\left(\begin{array}{ll}
1 & 1 \\
1 & 0
\end{array}\right)\left(\begin{array}{cc}
a_{2}-1 & 1 \\
1 & 0
\end{array}\right)
$$

provides

$$
\left[\overline{\left(a_{1},-1\right),\left(a_{2}, 1\right)}\right]=\left[\overline{a_{1}-1,1, a_{2}-1}\right]=a_{1}-1+\frac{1}{1+\frac{1}{a_{2}-1+\frac{1}{a_{1}-1+\ldots}}} .
$$

In particular $[\overline{(2,-1),(2,1)}]=[\overline{1}]=\frac{1+\sqrt{5}}{2}=G$. We have $\Omega(G)=\left(\begin{array}{ll}1 & 1 \\ 1 & 0\end{array}\right), \mathfrak{r}(\Omega(G))=G$, $\widetilde{\Omega}(G)=\Omega(G)^{2}$, and $\varrho(G)=4 \log (G)<\varrho_{E}(G)=4 \log (2+\sqrt{5})$. 
Example 4. $\omega=\left[\overline{\left(a_{1},-1\right),\left(a_{2},-1\right)}\right]=a_{1}-\frac{1}{a_{2}-\frac{1}{\omega}}$ with $a_{1} \neq a_{2}$ has minimal polynomial $\ell_{2} X^{2}-2 d \ell_{1} \ell_{2} X+\ell_{1}$ where $d:=\left(k_{1}, k_{2}\right), k_{1}=\ell_{1} d, k_{2}=\ell_{2} d,\left(\ell_{1}, \ell_{2}\right)=1$, and $\operatorname{disc}(\omega)=$ $4 \ell_{1} \ell_{2}\left(\ell_{1} \ell_{2} d^{2}-1\right)$.

The $E$-reduced QI $\omega=\ell_{1} d+\sqrt{\ell_{1}^{2} d^{2}-\frac{\ell_{1}}{\ell_{2}}}$ is not reduced as $\omega^{*}=\ell_{1} d-\sqrt{\ell_{1}^{2} d^{2}-\frac{\ell_{1}}{\ell_{2}}} \in(0,1)$.

The largest eigenvalue of $\Omega_{E}(\omega)=\left(\begin{array}{cc}a_{1} & -1 \\ 1 & 0\end{array}\right)\left(\begin{array}{cc}a_{2} & -1 \\ 1 & 0\end{array}\right)=\left(\begin{array}{cc}a_{1} a_{2}-1 & -a_{1} \\ a_{2} & -1\end{array}\right)$ is $\mathfrak{r}\left(\Omega_{E}(\omega)\right)=2 k_{1} k_{2}-1+$ $2 \sqrt{k_{1} k_{2}\left(k_{1} k_{2}-1\right)}$. Here $\widetilde{\Omega}_{E}(\omega)=\Omega_{E}(\omega)$ and so $\varrho_{E}(\omega)=2 \log \left(2 k_{1} k_{2}-1+2 \sqrt{k_{1} k_{2}\left(k_{1} k_{2}-1\right)}\right)$.

Example 5. $\omega=\llbracket a, \bar{b} \rrbracket=a-\frac{b}{2}+\frac{\sqrt{b^{2}-4}}{2}$ with $a \geq 2, b>2, a \neq b$ has minimal polynomial $X^{2}-(2 a-b) X+a^{2}-a b+1$. Then $\omega^{*}=a-\frac{b}{2}-\frac{\sqrt{b^{2}-4}}{2}$ and $\omega^{*} \notin(0,1)$, or else we get $b+\sqrt{b^{2}-4}<2 a<b+\sqrt{b^{2}-4}$, which yields $a=b$ - contradiction.

Example 6. The $B$-reduced QI $\omega=\llbracket \overline{3,6} \rrbracket=\frac{3+\sqrt{7}}{2}$ is not reduced as $\omega^{*} \notin(-1,0)$.

The largest eigenvalue of $\Omega_{B}(\omega)=\left(\begin{array}{cc}3 & -1 \\ 1 & 0\end{array}\right)\left(\begin{array}{cc}6 & -1 \\ 1 & 0\end{array}\right)=\left(\begin{array}{cc}17 & -3 \\ 6 & -1\end{array}\right)$ is $\mathfrak{r}\left(\Omega_{B}(\omega)\right)=8+3 \sqrt{7}$ and $\varrho_{B}(\omega)=2 \log (8+3 \sqrt{7})$. Note also that $\sqrt{7}=3-\frac{1}{\omega}=\llbracket 3, \overline{3,6} \rrbracket$.

\section{ACKNOWLEDGMENTS}

The research of the second author was partially supported by a 2020 University of Illinois Supplemental Summer Block Grant.

\section{REFERENCES}

[1] R. L. Adler, L. Flatto, The backward continued fraction map and the geodesic flow, Ergodic Theory Dynam. Systems 4 (1984), 487-492.

[2] V. Baladi, B. Vallée, Euclidean algorithms are Gaussian, J. Number Theory 110 (2005), 331-386.

[3] F. P. Boca, Products of matrices $\left[\begin{array}{ll}1 & 1 \\ 0 & 1\end{array}\right]$ and $\left[\begin{array}{ll}1 & 0 \\ 1 & 1\end{array}\right]$ and the distribution of reduced quadratic irrationals, J. Reine Angew. Mathematik 606 (2007), 149-165.

[4] F. P. Boca, R. N. Gologan, On the distribution of the free path length of the linear flow in a honeycomb, Ann. Inst. Fourier, 59 (2009), 1043-1075

[5] F. P. Boca, E. C. Merriman, Coding of geodesics on some modular surfaces and applications to odd and even continued fractions, Indagationes Math. 29 (2018), 1214-1234.

[6] F. P. Boca, J. Vandehey, On certain statistical properties of continued fractions with even and with odd partial quotients, Acta Arithmetica 156 (2012), 201-221.

[7] F. P. Boca, A. Zaharescu, On the correlations of directions in the Euclidean plane, Trans. Amer. Math. Soc. 358 (2006), 1797-1825.

[8] S. Cantrell, M. Pollicott, Comparison theorems for closed geodesics on negatively curved surfaces, preprint arXiv:2002.09767.

[9] F. Cellarosi, Renewal-type theorem for continued fractions with even partial quotients, Ergod. Theory Dynam. Syst. 29 (2009), 1451-1478.

[10] E. Cesaratto, B. Vallée, Gaussian behavior at quadratic irrationals, Acta Arithmetica 197 (2021), $159-205$.

[11] K. Dajani, D. Hensley, C. Kraaikamp, V. Masarotto, Arithmetic and ergodic properties of "flipped" continued fraction algorithms, Acta Arithmetica 153 (2012), 51-79.

[12] K. Dajani, C. Kraaikamp, "The mother of all continued fractions", Colloq. Math. 84/85 (2000), $109-123$.

[13] W. Duke, Hyperbolic distribution problems and half-integral weight Maass forms, Inventiones Math. 92 (1988), 73-90.

[14] C. Faivre, Distribution of Lévy constants for quadratic numbers, Acta Arithmetica 61 (1992), 13-34.

[15] E. Galois, Analyse algébrique. Démonstration d'un théorème sur les fractions continues périodiques, Ann. Math. Pures Appl. 19 (1828/29), 294-301.

[16] F. Halter-Koch, Quadratic irrationals: an introduction to classical number theory, CRC Press, 2013.

[17] B. Heersink, Distribution of the periodic points of the Farey map (with an appendix by F. P. Boca, B. Heersink and C. Merriman), Comm. Math. Phys. 365 (2019), 971-1003.

[18] F. Hirzebruch, Hilbert modular surfaces, Enseign. Math. 19 (1973), 183-281.

[19] J. Kallies, A. Özlük, M. Peter, C. Snyder, On asymptotic properties of a number theoretic function arising out of a spin chain model in statistical mechanics, Comm. Math. Phys. 222 (2001), 9-43. 
[20] D. Kelmer, Quadratic irrationals and linking numbers of modular knots, J. Modern Dynamics 6 (2012), 539-561.

[21] M. Kesseböhmer, S. Munday, B. Stratmann, Infinite ergodic theory of numbers, De Gruyter, 2016.

[22] C. Kraaikamp, A. O. Lopes, The theta group and the continued fraction expansion with even partial quotients, Geom. Dedicata 59 (1996), 293-333.

[23] D. Mayer, On a $\zeta$ function related to the continued fraction transformation, Bull. Soc. Math. France 104 (1976), 195-203.

[24] C. Merriman, Geodesic flows and the mother of all continued fractions, preprint arXiv:2001.06073

[25] T. Ono, An introduction to algebraic number theory, Plenum Press, 1990.

[26] G. Panti, A general Lagrange theorem, Amer. Math. Monthly 116 (2009), 70-74.

[27] M. Pollicott, Distribution of closed geodesics on the modular surface and quadratic irrationals, Bull. Soc. Math. France 114 (1986), 431-446.

[28] M. Pollicott, M. Urbanski, Asymptotic counting in conformal dynamics, preprint arXiv:1704.06896, to appear in Mem. Amer. Math. Soc.

[29] A. Rényi, Valós számok elöálli zsolgáló algoritmusokról., M. T. A. Mat. és Fiz. Oszt. Közl. 7 (1957), 265-293.

[30] F. Schweiger, Continued fractions with odd and even partial quotients, Arbeitsberichte Math. Institut Universität Salzburg 4 (1982), 59-70.

[31] F. Schweiger, On the approximation by continued fractions with odd and even partial quotients, Arbeitsberichte Math. Institut Universität Salzburg 1-2 (1984), 105-114.

[32] F. Schweiger, Numbertheoretical endomorphisms with $\sigma$-finite invariant measure, Israel J. Math. 21 (1975), 308-318.

[33] C. Series, The modular surface and continued fractions, J. London Math. Soc. 31 (1985), 69-80.

[34] H. J. S. Smith, Note on the theory of the Pellian equation and of binary quadratic forms of a positive determinant, Proc. London Math. Soc. s1-7 (1875), 196-208.

[35] M. Technau, The Calkin-Wilf tree and a trace condition, Master's Thesis, University of Würzburg, 2015.

[36] A. V. Ustinov, Spin chains and Arnold's problem on the Gauss-Kuzmin statistics for quadratic irrationals, Sbornik: Mathematics 204 (2013), 762-779.

[37] B. Vallée, Dynamical analysis of a class of Euclidean algorithms, Theoretical Computer Science 297 (2003), 447-486.

[38] B. Vallée, Euclidean dynamics, Discrete and Continuous Dynamical Systems 15 (2006), 281-352.

[39] D. Zagier, A Kronecker limit formula for real quadratic fields, Math. Ann. 213 (1975), 153-184.

Department of Mathematics, University of Illinois at Urbana-Champaign, Urbana, IL 61801

E-MAIL: FBOCA@ILLINOIS.EDU SISKAKI2@ILLINOIS.EDU 